\title{
Passive seismic monitoring with nonstationary noise sources
}

\section{Journal Article}

Author(s):

Delaney, Evan; Ermert, Laura; Sager, Korbinian; Kritski, Alexander; Bussat, Sascha; Fichtner, Andreas

Publication date:

2017-07

Permanent link:

https://doi.org/10.3929/ethz-b-000183055

Rights / license:

In Copyright - Non-Commercial Use Permitted

Originally published in:

Geophysics 82(4), https://doi.org/10.1190/geo2016-0330.1

Funding acknowledgement:

149143 - Full waveform ambient seismic noise inversion (SNF) 


\title{
Passive seismic monitoring with non-stationary noise sources
}

\author{
Evan Delaney*, Laura Ermert ${ }^{\dagger}$, Korbinian Sager $^{\dagger}$, Alexander Kritski ${ }^{\ddagger}$, Sascha \\ Bussat $^{\S}$, Andreas Fichtner ${ }^{\dagger}$ \\ *formerly TU Delft, Delft, The Netherlands, ETH Zürich, Zürich, Switzerland, and RWTH \\ Aachen, Aachen, Germany; currently Statoil, Stavanger, Norway. evde@statoil.com \\ ${ }^{\dagger}$ Institute of Geophysics, ETH Zürich, Zürich, Switzerland. \\ ${ }^{\ddagger}$ Statoil, Trondheim, Norway. \\ $\S$ Statoil, Bergen, Norway.
}

(February 8, 2017)

Running head: Passive monitoring with non-stationary noise

\begin{abstract}
Heterogeneous, non-stationary noise sources can cause traveltime errors in noise-based seismic monitoring. The effect worsens with increasing temporal resolution. This may lead to costly false alarms in response to safety concerns and limit our confidence in the results when these systems are used for quasi real-time monitoring of subsurface changes. We therefore develop a new method to quantify and correct these traveltime errors to more accurately monitor subsurface conditions at daily or even hourly timescales. This is based on the inversion of noise correlation asymmetries for the time-dependent distribution of noise sources. The source model is then used to simulate time-dependent ambient noise correlations. The comparison to correlations computed for homogeneous noise sources yields
\end{abstract}


traveltime errors that translate into spurious changes of the subsurface. The application of our method to data acquired at Statoil's SWIM array, a permanent seismic installation at the Oseberg field, demonstrates that fluctuations in the noise source distribution may induce apparent velocity changes of $0.25 \%$ within one day. Such biases thereby likely mask realistic subsurface variations expected on these timescales. These errors are systematic, dependent primarily on the noise source location and strength, and not on inter-station distance. Our method can then be used to correct for source-induced traveltime errors by subtracting these quantified biases in either data or model space. It can furthermore establish a minimum threshold for which we may reliably attribute traveltime changes to actual subsurface changes, should we not correct for these errors. In addition to the aforementioned real data scenario, we apply our method to a synthetic case for a daily passive monitoring overburden feasibility study. 


\section{INTRODUCTION}

\section{Passive monitoring using ambient noise}

Though its first successful application begins on the Sun (e.g. Duvall et al., 1993; Woodard, 1997; Rickett and Claerbout, 1999), ambient noise tomography has become a standard tool for imaging Earth structure on local, regional, and global scales (e.g. Sabra et al., 2005; Shapiro et al., 2005; Larose et al., 2006; Lin et al., 2008; Ruigrok et al., 2008; Nishida and Montagner, 2009; Bussat and Kugler, 2011; Verbeke et al., 2012; Boué et al., 2013; Nakata et al., 2015; Haned et al., 2016). The omnipresence of the ambient noise field makes this method well-suited for the monitoring of time-lapse subsurface changes beneath volcanoes (e.g. Brenguier et al., 2008b; Obermann et al., 2013), along active seismic faults (e.g. Brenguier et al., 2008a; Obermann et al., 2014), in hydrocarbon reservoirs (e.g. Bakulin et al., 2004; de Ridder and Biondi, 2013; Mordret et al., 2014b; de Ridder and Biondi, 2015), and within the vicinity of geothermal stimulation sites (e.g. Hillers et al., 2015; Obermann et al., 2015).

Most ambient noise tomographies rest on the approximation of an inter-station Green's function by the crosscorrelation of noise recorded at two stations. Theoretical requirements for Green's function retrieval include the homogeneous distribution of uncorrelated noise sources, the equipartitioning of the ambient noise field, and the absence of attenuation (e.g. Lobkis and Weaver, 2001; Weaver and Lobkis, 2001; Malcolm et al., 2004; Weaver and Lobkis, 2004; Curtis et al., 2006; Wapenaar and Fokkema, 2006; Wapenaar et al., 2006). While Lawrence and Prieto (2011) and Lawrence et al. (2013) suggest that attenuation may be included when azimuthally well-distributed noise sources are present, their result has been contested by the analytical work of Tsai (2011). 
In the Earth, the conditions for perfect Green's function retrieval are not satisfied. The Earth has spatially variable scattering properties and locally strong attenuation, especially in the near-surface, that prevent equipartitioning on a wide range of scales (e.g. Mulgaria, 2012; Sens-Schoenfelder et al., 2015). Furthermore, noise sources at any frequency are heterogeneously distributed and non-stationary (e.g. Peterson, 1993; Schulte-Pelkum et al., 2004; Bonnefoy-Claudet et al., 2006; Stehly et al., 2006; Tanimoto et al., 2006; Stutzmann et al., 2012; Latorre et al., 2014; Ermert et al., 2016). The detrimental effects on Green's function retrieval include incorrect traveltimes and amplitudes (Tsai, 2009; Froment et al., 2010; Cupillard and Capdeville, 2010; Tsai, 2011; Fichtner, 2014) and the appearance of spurious arrivals that largely preclude the use of higher-mode surface waves in noise correlations (Halliday and Curtis, 2008; Kimman and Trampert, 2010). To some extent, Green's function retrieval may be improved by processing (e.g. Bensen et al., 2007; Schimmel et al., 2011; Groos et al., 2012), but the procedures are subjective and lead to hardly predictable effects on the sensitivity of noise correlations to Earth structure (Fichtner, 2014).

\section{Motivation: Limitations caused by heterogeneous, non-stationary noise sources}

While traveltime errors induced by the heterogeneity of noise sources are often small enough for time-independent tomography (Froment et al., 2010), the non-stationarity of noise sources may translate into spurious time-lapse changes of subsurface structure. Indeed, Bussat (2015) presented an example where $5 \mathrm{~Hz}$ Scholte waves undergo a $2 \%$ shear wave phase velocity change in 4 hours - unrelated to subsurface production activity, but instead associated to a passing storm. 
To reduce such artifacts, two different approaches are typically followed: either the coda of the correlations is used under the assumption that multiple-scattering reduces the influence of the noise source direction (e.g. Brenguier et al., 2008b,a; Obermann et al., 2013,2014 ) or the averaging interval of the noise correlations is increased until noise source fluctuations are believed to average out to potentially recover surface and body waves (e.g. Claerbout, 1968; Bakulin et al., 2004; Bensen et al., 2007; de Ridder et al., 2014; Mordret et al., 2014b). While coda waves have been employed successfully in monitoring, their use is often not an option either due to the lack of sufficiently strong scatterers or to attenuation which may prevent their clear observation. This aspect is particularly important in nearsurface applications where $Q$ can be on the order of 10, thus preventing the emergence of coda.

In the context of hydrocarbon and geothermal reservoirs, the required time resolution is dictated by the timescales over which serious incidents may evolve; i.e., a few hours or days. Concrete applications include detecting - and preferably preventing - out-of-zone injections (e.g. Verdon et al., 2011), monitoring slop (produced water) and cuttings injectors, overseeing carbon sequestration sites (e.g. Eiken et al., 2011; Raknes et al., 2015), and optimizing future production targets by corroborating changes in pressure and production history profiles when current wells undergo shut-in tests and when new producers and injectors are brought online.

Since the extent to which source-related limitations are practically relevant is likely to be application-dependent, we consider the specific case of Statoil's SWIM (Seismic Waste Injection Monitoring) array, located above the Oseberg field off the Norwegian coast (Figure 1), and we aim to answer the following questions: 
(i) Within the frequency band $0.55-1.55 \mathrm{~Hz}$, do noise source distributions remain the same on daily timescales - in terms of both azimuthal coverage, strength, and frequency? (ii) If not, how much can noise sources fluctuate from day to day? (iii) What traveltime errors do we produce when we wrongly assume that correlations equal Green's functions?

(iv) What is the potential magnitude of the imaged changes in apparent velocity structure from day to day due to time-variable noise sources? (v) Are these artifacts sufficiently significant as to mask true variations of the subsurface? (vi) If they are, how can we remove them and further maximize our time resolution?

\section{Outline}

This paper is the first real data application of theory established in Hanasoge (2013) and extends upon this via methods found in Ermert et al. (2016). It is organized as follows: First, we compute daily stacks of noise correlations. Using measurements of correlation asymmetry, we invert for the power-spectral density distribution of the noise sources on a daily basis. With this realistic picture of noise sources and their temporal variability, we perform a series of synthetic inversions for $2 \mathrm{D}$ velocity structure: As artificial data, we use correlation functions computed for a homogeneous Earth model and the heterogeneous noise source distribution of a specific day. For the computation of synthetic data, we use the same homogeneous Earth model and a noise source distribution that is homogeneous as well. While the Earth model used to compute artificial and synthetic data is identical, the different distributions of noise sources induce systematic traveltime differences. Wave equation traveltime inversion then allows us to translate these traveltime differences into 2D maps of apparent velocity. The temporal variability of these maps provides a measure of apparent subsurface changes caused by the non-stationarity of the heterogeneous noise 
source distribution. Finally, instead of blindly performing waveform inversion with these biased traveltimes, we formulate how to remove these errors so that our velocities are unaffected by noise source non-stationarity. Preferably, this is done in the data space before proceeding to the tomography. We now discuss these steps in further detail.

\section{DATA: AMBIENT NOISE CORRELATIONS AT THE SWIM ARRAY}

\section{Acquisition layout, data characteristics, and processing}

The SWIM array, shown in Figure 1, consists of 172 four-component receivers (MEMS accelerometer and hydrophone) linked through a single ocean-bottom cable. The configuration lies along the ocean floor, roughly $108 \mathrm{~m}$ below mean sea level, about $150 \mathrm{~m}$ south of the Oseberg C platform. Its purpose is to monitor cuttings injected into the overburden. Receiver spacing is $\sim 50 \mathrm{~m}$ for receivers on the outside boundaries of the array and reduces to $\sim 25 \mathrm{~m}$ for receivers in its inner portion. The spread of the array's outer right-side is around $1.74 \mathrm{~km}$ and the horizontal separation of the right-side's inner and outside segments halfway down the array is approximately $300 \mathrm{~m}$. Similar properties apply to its left-side. Further information about its layout can be found in Bussat (2015).

The frequency response ranges from direct current, used to measure the gravity vector, to beyond $200 \mathrm{~Hz}$. All receivers operate continuously, sampling at $500 \mathrm{~Hz}$. The accessible data ranges from April 30, 2014 to May 18, 2015 and totals about 40 TB.

Above $1.55 \mathrm{~Hz}$, we observe strong noise originating from the platform. Since noise at higher frequencies is most likely anthropogenic (e.g. Bonnefoy-Claudet et al., 2006) and - in our case - predominantly spatially stationary, we limit our study to frequencies of $1.55 \mathrm{~Hz}$ and below. We also do not study frequencies below $0.55 \mathrm{~Hz}$, as our method requires that 
causal and acausal arrivals be clearly distinguishable.

To compute inter-station correlation functions, we first divide the data for one day into $225 \mathrm{~s}$ segments. For each segment, we rotate the data to correct for misorientation, extract the vertical component, lowpass filter to $10 \mathrm{~Hz}$, downsample to $20 \mathrm{~Hz}$, and compute geometrically normalized crosscorrelations for all possible receiver pairs (e.g. Schimmel, 1999; Seats

et al., 2012). We then stack all segments to obtain the averaged correlations for one day. Even though we do not expect to observe waves taking more than $10 \mathrm{~s}$ to propagate across an array with $<1.8 \mathrm{~km}$ maximum offset, we lag the correlations up to $30 \mathrm{~s}$. This allows us to estimate the signal-to-noise ratio and reject correlations without clear signals. We deliberately do not apply non-linear processing techniques, such as spectral whitening or one-bit normalization (Bensen et al., 2007; Groos et al., 2012), even though the latter has been shown to not add a systematic bias to the measurement in the case of Gaussian wavefield statistics and for a variety of other distributions (Hanasoge and Branicki, 2013; Fichtner et al., 2016). This is intended to avoid non-physical biases in the correlations (Fichtner, 2014) and to preserve valuable information on the distribution of the noise sources.

\section{Spatio-temporal variability}

Figure 2 shows a selection of correlation functions for different receiver-receiver azimuths and for two different days: July 21 and July 22, 2014. The correlation functions are dominated by large-amplitude Scholte wave arrivals with a group velocity of $\sim 300 \mathrm{~m} / \mathrm{s}$. All examples exhibit a pronounced asymmetry that varies with both azimuth and time, thus attesting to the spatio-temporal variability of the underlying noise sources. 


\section{THE POWER-SPECTRAL DENSITY DISTRIBUTION OF NOISE SOURCES}

\section{Modeling inter-station correlations}

To estimate the distribution of noise sources quantitatively in space and time, we start with the formulation of a forward model that allows us to compute synthetic correlation functions for a predefined noise source. Since our focus is on horizontally propagating Scholte waves in vertical-component correlations, we adopt the acoustic approximation of the frequency-domain representation theorem

$$
u(\mathbf{x}, \omega)=\int G(\mathbf{x}, \boldsymbol{\xi}, \omega) N(\boldsymbol{\xi}, \omega) d^{2} \boldsymbol{\xi}
$$

where $u(\mathbf{x}, \omega)$ is the seismic wavefield at position $\mathbf{x}, \omega$ denotes the circular frequency, $N(\boldsymbol{\xi}, \omega)$ is the noise source force density at position $\boldsymbol{\xi}$, and $G(\mathbf{x}, \boldsymbol{\xi}, \omega)$ is the Green's function of the medium that relates the noise source to the wavefield. Based on equation (1), the interstation correlation $C\left(\mathbf{x}_{i}, \mathbf{x}_{k}\right)=u\left(\mathbf{x}_{i}\right) u^{*}\left(\mathbf{x}_{k}\right)$ takes the form

$$
C\left(\mathbf{x}_{i}, \mathbf{x}_{k}\right)=\iint G\left(\mathbf{x}_{i}, \boldsymbol{\xi}\right) G^{*}\left(\mathbf{x}_{k}, \boldsymbol{\xi}^{\prime}\right) N(\boldsymbol{\xi}) N^{*}\left(\boldsymbol{\xi}^{\prime}\right) d^{2} \boldsymbol{\xi} d^{2} \boldsymbol{\xi}^{\prime}
$$

where we omit the dependence on $\omega$ in the interest of a condensed notation. Ambient noise in the oceans at frequencies between $0.5-2.0 \mathrm{~Hz}$ is most likely caused by the interaction of wind-waves with wavelengths below $25 \mathrm{~m}$ (Gimbert and Tsai, 2015). We can therefore assume that the temporal correlation of neighboring noise sources $N(\boldsymbol{\xi}) N^{*}\left(\boldsymbol{\xi}^{\prime}\right)$ in equation (2) is a $\delta$-function compared to the seismic wavelength that exceeds several hundred meters.

This widely used approximation (e.g. Woodard, 1997; Hanasoge, 2013, 2012; Fichtner, 2014; Nishida, 2014) simplifies the inter-station correlation to

$$
C\left(\mathbf{x}_{i}, \mathbf{x}_{k}\right)=\int G\left(\mathbf{x}_{i}, \boldsymbol{\xi}\right) G^{*}\left(\mathbf{x}_{k}, \boldsymbol{\xi}\right) S(\boldsymbol{\xi}) d^{2} \boldsymbol{\xi}
$$


where $S(\boldsymbol{\xi})=N(\boldsymbol{\xi}) N^{*}(\boldsymbol{\xi})$ is the power-spectral density ( $\left.p s d\right)$ distribution of the noise sources as a function of space and frequency. Equation (3) constitutes a forward modeling equation that allows us to directly compute synthetic inter-station correlations from the deterministic quantity $S(\boldsymbol{\xi})$, without the need to model a quasi-random wavefield excited by stochastic sources.

\section{Measuring causal-to-acausal energy ratios}

To extract robust information on the psd of the noise sources, we follow the approach of Ermert et al. (2016) and measure causal-to-acausal energy ratios in the daily correlations, defined as

$$
A=\ln \frac{\int\left[w_{+}(t) \bar{C}(t)\right]^{2} d t}{\int\left[w_{-}(t) \bar{C}(t)\right]^{2} d t}=\ln \frac{E_{+}}{E_{-}}
$$

where $A$ is the correlation asymmetry; $\bar{C}(t)$ is the time-domain version of $C(\omega) ; w_{+}(t)$ represents the window centered around the causal wave of $\bar{C}(t)$; and $w_{-}(t)$ signifies the window centered around the acausal wave; $E_{+}$and $E_{-}$denote the energy of the causal and acausal wave packets under $w_{+}(t)$ and $w_{-}(t)$, respectively. The measurement process is illustrated in Figure 3 for receiver pair 36-145 that shows clear causal and acausal Scholte wave arrivals.

The amplitude ratio equals 0 in the case of a symmetric correlation function, thus indicating that noise source energy first recorded by an inter-station pair's first receiver which later propagates onward to the second receiver is the same as the energy first arriving at the second receiver which then propagates towards the first receiver. However, when $A<0$, the acausal amplitudes are larger than those on the causal side, as observed for instance in Figure 3. This signifies that the source strength on the side of the first receiver 
(receiver 36 in our example) is stronger than that on the side of the second receiver.

The measurement of amplitude ratios has the advantage of being primarily sensitive to the noise source distribution, while being practically insensitive to visco-elastic attenuation (Lawrence and Prieto, 2011) and focusing induced by unknown Earth structure.

\section{Sensitivity kernels for the noise source $p s d$}

To solve an inverse problem for the $p s d$ of the noise sources, we compute synthetic correlation functions using the forward modeling equation (3). As an initial noise source model, we use an annulus of constant psd $S(\boldsymbol{\xi})$, as shown in the background of Figure 4 . We then quantify the difference between the synthetic correlation asymmetry $A_{i k}$ and the observed asymmetry $A_{i k}^{\mathrm{o}}$ using the $L_{2}$ misfit functional

$$
\chi=\frac{1}{2} \sum_{i k}\left(A_{i k}-A_{i k}^{\mathrm{o}}\right)^{2},
$$

where the subscripts $i$ and $k$ respectively denote the receivers in a receiver pair. Using adjoint techniques (e.g. Tarantola, 1988; Plessix, 2006; Fichtner et al., 2006; Fichtner, 2010) we can compute sensitivity kernels of the misfit $\chi$ with respect to the noise source $p s d$ (e.g. Tromp et al., 2010; Hanasoge, 2012; Fichtner, 2014; Ermert et al., 2016).

Figure 4a shows the finite-frequency noise source kernel for station pair 36-145, already used in the example of Figure 3. The kernel indicates where the noise source $p s d$ should be increased (darker colors) and where it should be decreased (lighter colors), relative to the azimuthally homogeneous initial psd (background annulus).

To reduce the computational costs of noise source inversion, we adopt the ray-theoretical simplification of the noise source kernels proposed by Ermert et al. (2016). The raytheoretical kernel corresponding to the finite-frequency kernel in Figure 4a, is shown below 
in Figure 4b. The simplified kernels allow us to obtain images of noise sources with negligible computational effort, which is particularly beneficial for noise source imaging on a daily basis or even shorter time intervals.

As shown by Ermert et al. (2016), kernel-based noise source inversion leads to results that are qualitatively similar to beamforming, in the sense that nearly identical source regions are mapped by both methods. This is to be expected because sensitivity kernels roughly act as beams pointing in the direction of possible noise sources, and with strength proportional to the noise source amplitudes. The outstanding advantage of our inversion over beamforming is that it provides the actual psd of the noise sources with proper physical units $\left(\mathrm{m}^{-4} \mathrm{~N}^{4} \mathrm{~s}^{2}\right)$, needed to solve the forward modelling equation (3).

\section{Noise sources on July 21 and July 22, 2014}

While we analyze data for a complete year, we limit ourselves to the presentation of results for July 21 and July 22, 2014, in the frequency band 1.15-1.55 Hz. This is sufficient to demonstrate the concept. However, we have also generated noise source sensitivity kernels for all other days from April 30, 2014 to May 18, 2015, in the frequency bands 0.55-0.95 $\mathrm{Hz}, 0.75-1.15 \mathrm{~Hz}$, and $0.95-1.35 \mathrm{~Hz}$. These can be found in the electronic supplement. The choice of these two days is that July 21 and 22 are good representatives of the complete dataset, as there are days when noise sources change less, and other days when they change more.

Though 172 receivers yield 14706 correlation functions, we limit our analysis to station pairs with an offset that is sufficiently large to clearly distinguish causal and acausal arrivals. For the frequency band $1.15-1.55 \mathrm{~Hz}$, we therefore use offsets $\geqslant 855.2 \mathrm{~m}$, which leaves 4190 
station pairs.

For both days, July 21 and July 22, 2014, we reject few station pairs with poor signal-tonoise ratio. This further reduces the number of correlations to 4144 and 4054, respectively. The final azimuthal coverage, shown in Figure 5, is very similar for both days. Nonetheless, we bin and weight the sensitivity kernels appropriately (i.e., by dividing each bin's cumulative kernel sum by its fold to produce its kernel average) so that all azimuths are treated equally. This ensures that any changes observed in the noise source kernels are not biased by data selection.

The cumulative noise source kernels of all receiver pairs for July 21 and July 22, 2014, are displayed in Figure 6. Prior to an actual inversion for the noise source $p s d$, these kernels provide an initial image of the noise sources and temporal variability. On July 21, 2014, noise sources west of the SWIM array were stronger than average, and weaker than average to the east. On July 22, 2014, the distribution has changed, and stronger than average noise sources are now located to the north.

The sensitivity kernels indicate that only azimuthal variations of the noise source psd can be constrained. This justifies the inversion for the $p s d$ distribution along an annulus surrounding the array. For this inversion, we use the previously computed kernels in a steepest-descent minimization of the misfit defined in equation (5). While the convexity of a misfit functional, required to ensure convergence towards the global optimum, is generally difficult to assess, there are at least plausibility arguments: (i) Since we measure waveform energies, our misfit does not suffer from cycle skipping. This major source of non-linearity and non-convexity is therefore automatically eliminated. (ii) The steplength tests performed in this study show that misfit generally increases away from the optimal steplength. This 
indicates that the misfit is convex at least along selected directions. The optimal psd distributions for July 21 and July 22, 2014, are shown in Figure 7.

\section{APPARENT TRAVELTIME DIFFERENCES AND EARTH STRUCTURE INDUCED BY HETEROGENEOUS NOISE SOURCES}

\section{Systematic traveltime biases}

The SWIM array data provide us with realistic distributions of the noise source psd in the North Sea surrounding the array on a daily basis. In the following, we will use these in numerical experiments intended to quantify systematic traveltime biases and apparent subsurface structure.

Our modeling is based on the time-domain finite-difference solution of the acoustic wave equation (Virieux, 1984) with absorbing boundary conditions (Cerjan et al., 1985). Using an acoustic approximation as an analogue for Scholte wave propagation is computationally efficient and appropriate for narrow frequency bands where dispersion can be ignored. We choose a homogeneous Earth model $\mathbf{m}$ that permits waves to propagate at $300 \mathrm{~m} / \mathrm{s}$, which corresponds to the average group velocity of Scholte waves recorded by the SWIM array at frequencies between 1.15-1.55 Hz.

Using the psd distributions of July 21 and July 22, 2014 (Figure 7), we compute timedomain inter-station correlations $\bar{C}^{\mathrm{o}}\left(\mathbf{x}_{i}, \mathbf{x}_{k}, t\right)$ by solving the forward modeling equation (3). We treat these correlation functions as artificial data. Emulating the common practice in ambient noise interferometry that assumes that noise sources are favorably distributed to enable Green's function recovery (e.g. Sabra et al., 2005; Shapiro et al., 2005; Mordret et al., 2014a; de Ridder and Biondi, 2015), we then compute synthetic correlation functions 
$\bar{C}\left(\mathbf{x}_{i}, \mathbf{x}_{k}, t\right)$ using an isotropic psd distribution.

To quantify the difference between traveltimes for the heterogeneous $p s d, \mathcal{T}_{i k}^{\mathrm{o}}(\mathbf{m})$, and the homogeneous $p s d, \mathcal{T}_{i k}(\mathbf{m})$, we measure the time where the cross-correlation between the synthetics $\bar{C}\left(\mathbf{x}_{i}, \mathbf{x}_{k}, t\right)$ and the artificial data $\bar{C}^{\mathrm{o}}\left(\mathbf{x}_{i}, \mathbf{x}_{k}, t\right)$ attains its global maximum (e.g. Luo and Schuster, 1991; Dahlen et al., 2000),

$$
\Delta \mathcal{T}_{i k}(\mathbf{m})=\arg \max \int \bar{C}^{\mathrm{o}}\left(\mathbf{x}_{i}, \mathbf{x}_{k}, t\right) \bar{C}\left(\mathbf{x}_{i}, \mathbf{x}_{k}, t+\tau\right) d t
$$

The traveltime difference $\mathcal{T}_{i k}(\mathbf{m})$ is negative when the synthetic $\bar{C}\left(\mathbf{x}_{i}, \mathbf{x}_{k}, t\right)$ arrives earlier than the artificial data $\bar{C}^{\mathrm{o}}\left(\mathbf{x}_{i}, \mathbf{x}_{k}, t\right)$, and vice versa.

Figure 8 displays $\mathcal{T}_{i k}(\mathbf{m})$ as a function of receiver pair azimuth for July 21 and July 22, 2014. Traveltime differences oscillate between -10 and $10 \mathrm{~ms}$ with a quasi-period of $\sim 30^{\circ}$. Notable differences on the order of $5 \mathrm{~ms}$ exist between the two days. Since both artificial data and synthetics have been computed for the same homogeneous Earth model, the traveltime differences represent a systematic error induced by the heterogeneous distribution of noise sources. .

\section{Static apparent Earth structure}

As shown in Figure 8, average traveltime differences of $\sim 5 \mathrm{~ms}$ can be observed over an average inter-station distance of $\sim 1000 \mathrm{~m}$ in the SWIM array. This translates into apparent velocity variations of $\sim 0.5 \mathrm{~m} / \mathrm{s}$ spread over the complete inter-station distance.

To quantify the spatial distribution of apparent velocity heterogeneities induced by the heterogeneous noise sources, we invert the apparent traveltimes from Figure 8 for a 2D group velocity model. In order to be consistent with the finite-frequency traveltime measurements and to avoid additional systematic errors that may be introduced by the ray approximation 
(e.g. Wielandt, 1987; Montelli et al., 2004; Malcolm and Trampert, 2011), we perform nonlinear wave equation traveltime tomography, as introduced by Luo and Schuster (1991). The resulting velocity models are displayed in Figure 9.

For both days, lateral velocity variations of $\pm 1.0 \mathrm{~m} / \mathrm{s}$ are required to optimally explain the traveltime differences. There is no obvious large-scale pattern that may be related intuitively to the noise source distribution. Heterogeneities outside of the array are caused by the spatially extended finite-frequency kernels used in the wave-equation traveltime inversion.

\section{Apparent subsurface changes}

The apparent velocity heterogeneities on July 21 and July 22 differ markedly, thus giving the incorrect impression of subsurface changes. The distribution and magnitude of the apparent velocity change is displayed in Figure 10, which shows the difference between the velocity maps in Figure 9. Average velocity changes are approximately $\pm 0.5 \mathrm{~m} / \mathrm{s}$, but local extrema exceed $\pm 0.75 \mathrm{~m} / \mathrm{s}$. These apparent variations of subsurface structure from one day to the next are solely the result of rapidly moving noise sources. If we had not known these changes were due to non-stationary noise sources, we would wrongly interpret these as subsurface changes.

\section{REMOVAL OF SYSTEMATIC ERRORS IN NOISE-BASED MONITORING}

The sequence of methods introduced in the previous sections - from noise source imaging to finite-frequency traveltime tomography - can be used constructively to correct for systematic 
traveltime and velocity errors. This is achieved by subtracting these quantified errors from either the data or model space.

\section{Correcting systematic biases}

To minimize the contamination of velocity maps that results from heterogeneously distributed noise sources, the apparent traveltime differences $\Delta \mathcal{T}_{i k}(\mathbf{m})$ computed for an Earth model $\mathbf{m}$

$$
\Delta \mathcal{T}_{i k}(\mathbf{m})=\mathcal{T}_{i k}(\mathbf{m})-\mathcal{T}_{i k}^{\mathrm{o}}(\mathbf{m})
$$

introduced in equation (6) and shown in Figure 8, can be subtracted from the total observed traveltime differences

$$
\Delta T_{i k}(\mathbf{m})=\mathcal{T}_{i k}(\mathbf{m})-T_{i k}^{\mathrm{o}}
$$

where $T_{i k}^{o}$ is the traveltime of the observed noise correlation waveform, $\mathcal{T}_{i k}(\mathbf{m})$ is the traveltime of synthetics with homogeneous $p s d$, and $\mathcal{T}_{i k}^{\mathrm{o}}(\mathbf{m})$ is the traveltime of synthetics with the heterogeneous psd inferred from the data. The difference of $\Delta T_{i k}(\mathbf{m})$ and $\Delta \mathcal{T}_{i k}(\mathbf{m})$ eliminates the traveltime of the synthetic correlation waveform with homogeneous source distribution $\mathcal{T}_{i k}(\mathbf{m})$ and yields the source-corrected traveltime difference

$$
\Delta T_{i k}^{\text {corr. }}(\mathbf{m})=\Delta T_{i k}(\mathbf{m})-\Delta \mathcal{T}_{i k}(\mathbf{m})=\mathcal{T}_{i k}^{\mathrm{o}}(\mathbf{m})-T_{i k}^{\mathrm{o}}
$$

Equation (9) gives the correct traveltime difference between synthetic noise correlation waveforms computed for an inferred heterogeneous source distribution and the observed noise correlation waveforms. It may thus be used to perform traditional ambient noise tomography where correlations are assumed to equal Green's functions, without the need to modify an existing inversion machinery. 
For consistency, the traveltime difference in equation (8) must be computed with the same method used to measure $\Delta \mathcal{T}_{i k}$; i.e., by crosscorrelation in our case. Since the apparent traveltime differences $\Delta \mathcal{T}_{i k}(\mathbf{m})$ have a second-order dependence on the underlying Earth model $\mathbf{m}$ (Fichtner, 2015; Ermert et al., 2016), they should ideally be updated regularly during an iterative inversion of the corrected traveltime differences $\Delta T_{i k}^{\text {corr. }}(\mathbf{m})$.

Instead of performing corrections in data space, corrections in model space can be an alternative. For instance, inferences of true changes in subsurface structure may be improved by subtracting apparent velocity changes. Furthermore, the maps of apparent velocity changes can be used to identify regions where we may safely interpret. However, as the systematic errors exhibit a strong angular dependence that no reasonable model could replicate, it is more advisable to remove these errors directly from the data space before proceeding to invert for structure. This is further addressed in a subsequent section: Analysis of noise source non-stationarity.

\section{Synthetic inversion example}

To illustrate the basic concept outlined above, we perform a synthetic numerical experiment using the SWIM array configuration. Unlike in the real-life case, we now have full control over the structure and noise perturbations from one day to the next. We now present three cases.

Case I - The baseline. On the first day, our structure is homogeneous and allows surface waves to propagate at $300 \mathrm{~m} / \mathrm{s}$. The noise source distribution is homogeneous as well. On the second day, our structure undergoes a regional perturbation of up to $0.3 \%$, as shown in Figure 11a. However, the noise source distribution also undergoes a perturbation on 
the second day and becomes inhomogeneous. The true model for the $p s d$ is a smoothed version of the imaged July 21 psd in Figure 7. Measuring the traveltime difference in the correlation functions between the first and second day, we perform a waveform inversion and image the structural perturbation, assuming perfect knowledge of the heterogeneous noise source distribution. The result is shown in Figure 11b. As a result of imperfect coverage, the target perturbation of Figure 11a is, of course, not perfectly reconstructed. However, since the heterogeneous noise sources are fully taken into account, all the differences between Figures $11 \mathrm{a}$ and $11 \mathrm{~b}$ are purely imaging artifacts. In this regard, we have produced the best possible reconstruction of the structural perturbations, given our source-receiver setup.

Case II - Ignoring non-stationary noise sources. Since the time-variable noise source distributions are in practice not known a priori. We therefore repeat the previous inversion, incorrectly assuming that the noise source distribution has remained homogeneous from day 1 to day 2. As shown in Figure 11c, the reconstructed anomaly is distorted substantially relative to the baseline of case I. Additional artifacts appear in the northwestern part of the model in the form of strong positive velocity changes. These artifacts are purely the result of the non-stationary noise sources that have not been taken into account.

Case III: We apply the method presented in this paper to correct for the non-stationary noise source traveltime biases. By taking the amplitude ratios of correlation functions from the second day, we then create a noise source kernel. Using a homogeneous structure model and a homogeneous noise source model, we then invert for the noise source psd. Using the imaged noise source $p s d$, we then determine the traveltime bias produced from the inhomogeneous noise source distribution. We subtract this from the traveltime differences in case II to yield corrected traveltimes. Inverting for these corrected traveltimes, we produce a corrected structure image, shown in Figure 11d. Since Figure 11d is nearly identical to 
the baseline in Figure Figure 11b, we conclude that the artifacts due to non-stationary noise sources have been removed almost completely.

While unavoidably simplistic in nature, our synthetic inversion illustrates that the proposed method is self-consistent, and that artifacts due to non-stationary noise sources may indeed be removed under favorable circumstances. Nonetheless, results for real data applications may be different based on the structure we wish to image, the quality of the data, the magnitude of the structural change versus the noise source psd change, etc. This is the subject of sensitivity studies, an example of which will be presented in the following paragraphs.

\section{Traveltime sensitivity and uncertainties}

The extent to which we can remove these traveltime artifacts depends upon the prediction error in the traveltime bias $\Delta \mathcal{T}_{i k}(\mathbf{m})$ that results from uncertainties in the inferred noise source model. These uncertainties are rooted in the measurement error, and would be smaller for measurements that are better in quality and coverage.

We estimate the prediction error through forward modelling with perturbed $p s d s$. For this, we perturb the optimal step length $\sigma_{\text {g.m. }}$ that minimizes the misfit to a perturbed step length $\sigma_{\text {pert }}$ until it produces synthetic amplitude ratios that differ from the observed amplitude ratios by more than one standard deviation of the measurement error. We then compute the traveltime correction factor from this maximally perturbed $p s d$. Comparing the traveltime correction factor of the perturbed $p s d, \Delta \mathcal{T}_{i k}\left(\mathbf{m}, \sigma_{\text {pert. }}\right)$, to that of the globally

minimized $p s d, \Delta \mathcal{T}_{i k}\left(\mathbf{m}, \sigma_{\text {g.m. }}\right)$, we can deduce a prediction error $\Delta \mathcal{T}_{i k}^{\text {err. }}\left(\mathbf{m}, \sigma_{\text {pert. }}, \sigma_{\text {g.m. }}\right)$ by taking the difference of $\Delta \mathcal{T}_{i k}\left(\mathbf{m}, \sigma_{\text {pert. }}\right)$ and $\Delta \mathcal{T}_{i k}\left(\mathbf{m}, \sigma_{\text {g.m. }}\right)$. 
Referring back to the SWIM array real data case, we can perturb the step length by $\sim 15 \%$ for both July 21 and July 22 . This results in a traveltime prediction error of $\sim \pm 1$ ms for July 21 and $\sim \pm 1.5 \mathrm{~ms}$ for July 22 , which translates to $\sim 10-20 \%$ uncertainty in the traveltime correction factor $\Delta \mathcal{T}_{i k}\left(\mathbf{m}, \sigma_{\mathrm{g} . \mathrm{m}}\right)$. Thus, noise source non-stationarity may induce apparent subsurface changes of up to $\sim 0.30 \%$ (and not $\sim 0.25 \%$ ), if we account for such uncertainty using this criterion. From July 21 to July 22, we may thus argue that we would not observe any apparent subsurface changes that are within $\sim 10-20 \%$ of our traveltime correction factor. Moreover, if we cannot readily update existing machinery, algorithms, or existing workflows to account for the quantities $\Delta \mathcal{T}_{i k}\left(\mathbf{m}, \sigma_{\text {g.m. }}\right)$ and $\Delta \mathcal{T}_{i k}^{\text {err. }}\left(\mathbf{m}, \sigma_{\text {pert. }}, \sigma_{\text {g.m. }}\right)$, we may instead set a minimum threshold (e.g., $\sim 0.30 \%$ apparent velocity structure change) at which we may observe subsurface changes and not attribute them to noise source non-stationarity. This minimum threshold will vary and depend upon the standard deviation of the amplitude ratio measurements and the heterogeneity of the noise source distribution - it is very application dependent. We direct the reader to the electronic supplement in the Appendix section for more information on the computation of the prediction errors.

\section{DISCUSSION}

\section{Comparison to velocity changes observed in previous studies}

To assess the significance of the apparent velocity variations shown in Figure 10, we summarize previous findings of time-variable subsurface structure. Using ambient noise tomography for the Valhall field, de Ridder et al. (2014) found velocity changes of $\sim 2 \%$ over 
a 6-year period within the same frequency band used here. Assuming a constant change over time, we translate this to an average daily velocity change of $10^{-3} \%$, i.e., 2 orders of magnitude less than the systematic errors observed at Oseberg from July 21 - 22, 2014. However, the authors make this 6 year difference map by choosing only 29 hours from February 2004 and 5 days from December 2010. Though they show that the correlation functions are stable within each of these periods, they do not guarantee that they were produced from the same noise source configuration. This may explain discrepancies between their velocity maps and those produced from an active survey. Moreover, Mordret et al. (2014a) determine yearly velocity variations (also from Valhall) that vary between $\pm 0.08 \%$ - less than those presented here. Again, these authors only use 29 hours from 2004 and 6.5 hours from 2005, which also suggests that noise source fluctuations could play an undesired role in these velocity variations.

Outside the context of hydrocarbon reservoir monitoring, time-lapse velocity changes of 0.01-1.0 \% over several weeks to years have been observed in relation to volcanic activity (Brenguier et al., 2008b; Obermann et al., 2013), large earthquakes (Brenguier et al., 2008a; Obermann et al., 2014), seasonal variations of the groundwater table (Meier et al., 2010), and the stimulation of geothermal reservoirs (Hillers et al., 2015; Obermann et al., 2015).

It follows that the apparent velocity changes induced by non-stationary noise sources are significant. They limit the quality and time resolution of ambient noise monitoring based on noise correlation surface waves and the traditional assumption of perfect Green's function recovery, unless corrections such as those proposed in the previous sections are applied. 


\section{Velocity changes deduced from noise correlation coda}

Instead of analyzing the fundamental-mode interface wave, several previous studies used noise correlation coda; assuming that multiple scattering makes it is less susceptible to noise source variations (e.g. Brenguier et al., 2008b; Meier et al., 2010; Hillers et al., 2015). However, as demonstrated by Zhan et al. (2013), coda waveforms are affected by changes in the frequency content of noise sources. Furthermore, clearly observable coda is not generally present, e.g. when attenuation is strong or when the medium is smooth. From a theoretical perspective, it is well known that only fundamental-mode waves can be reconstructed accurately by noise correlations (e.g. Halliday and Curtis, 2008; Kimman and Trampert, 2010). Unless noise sources are perfectly homogeneously distributed or the wavefield is equipartitioned - which is not the case in the Earth (e.g. Mulgaria, 2012; Sens-Schoenfelder

et al., 2015) - the actual physical nature of the noise correlation coda is therefore unclear. This complicates the imaging procedure. It follows from these arguments that the method presented here is more widely applicable, and it rests on a more solid theoretical foundation.

In the context of our specific dataset, we note that clear coda waves are not present in the noise correlations. This may be due to either insufficiently strong scatterers or attenuation, as discussed above.

\section{Nature of apparent traveltime differences}

We see from Figure 8 that apparent traveltime differences exhibit a strong azimuthal dependence with a quasi-period of $\sim 30^{\circ}$. On July 21, for instance, traveltimes differ by more than $20 \mathrm{~ms}$ for azimuths from $\sim 120^{\circ}$ to $\sim 150^{\circ}$. This suggests a form of anisotropy with an approximately 12 -fold symmetry axis that does not exist in minerals composing 
the Earth. The unphysical nature of the traveltime differences becomes evident in Figure 12. It reveals that traveltime differences do not increase systematically with offset, but decrease slightly. This can be interpreted in terms of sensitivity kernels: As the inter-station distance increases the noise source kernels become narrower, and thus the measurements are less sensitive to the source distribution. These traveltime errors are thus systematic, fundamentally linked to the power and azimuthal layout of the noise sources.

It may appear counterintuitive to have arrivals that occur later than the maximum arrival time expected when sources are located within the stationary-phase region (Snieder, 2004; Wapenaar et al., 2010). However, the stationary-phase region is not precisely defined, and finite-frequency sensitivity kernels of traveltimes with respect to noise sources for ballistic waves yields a kernel that has both positive and negative contributions. This indicates that finite-frequency traveltimes may indeed be larger and is a facet of apparent finite-frequency paradoxa.

This aspect is also considered by Tsai (2009), where the author analytically derives a negative-positive oscillatory nature of traveltime biases for finite-frequency cases even for an isotropic distribution of sources. In this case, noise source frequency, inter-station separation, and the velocity of the medium account for traveltime biases. As these parameters would most likely remain unchanged from day to day, it would then be a dynamic noise source distribution that would bring about the pattern that we observe in Figure 8.

The assumption that correlation functions equal Green's functions thus leads to unphysical traveltime measurements that can hardly be explained without unrealistic anisotropy or excessively heterogeneous Earth structure. In our traveltime inversions, we prevent the appearance of unresolvable sub-wavelength structure. As a consequence, we could only 
reduce the misfit by $\sim 25 \%$, starting from a homogeneous model on both July 21 and 22 .

When clearly visible, the unphysical nature of the traveltime differences may be used as an indicator of significant systematic errors induced by non-stationary noise sources. In the specific case of the SWIM array, the absence of an offset-dependence suggests that the errors may be reduced by using only the largest offsets.

\section{Analysis of noise source non-stationarity}

July 21 and July 22, 2014, are days on which the noise source azimuthal mean moves by $\sim 90^{\circ}$. While noise sources vary more slowly throughout most of the year, similarly strong daily variations are still not uncommon. This is shown most clearly in Figure 13 where we plot the noise source angular mean and the correlation functions' absolute energy ratio as a function of daily and likewise of monthly averaging within the frequency bands $0.55-0.95$ $\mathrm{Hz}$ and 1.15-1.55 Hz.

For daily averaging, we note that fluctuations in mean noise source position and $p s d$ (inferred via the energy ratio plot) can occur with little predicability. Indeed, while the 1.15-1.55 Hz daily angular mean from April 29, 2014 to May 18, 2015, is $\sim 215^{\circ}$, the mean experiences its maximum fluctuation from August 7 to August 8, 2014, shifting by $>210^{\circ}$. Furthermore, we observe that the energy ratio also changes rapidly from day to day. This indicates that not only do systematic errors map into different regions within the data and model space, but also with varying magnitudes. Moreover, these complications are further compounded if we decide to use different frequency bands: Whereas the angular mean of the sources within the $1.15-1.55 \mathrm{~Hz}$ band is stable in February, this does not hold for the 0.55-0.95 Hz band. Thus, to ensure reliable monitoring on a daily basis, methods that 
account for the non-stationary nature of noise sources must be implemented.

These issues mostly vanish once we choose to stack our correlations on monthly timeframes. Though the noise field remains inhomogeneous and artifacts leak into static traveltime measurements, these cancel out when determining the dynamic traveltime differences from month to month. A caveat about this is that the energy ratio changes per month notably within the $0.55-0.95 \mathrm{~Hz}$ band, where we witness a $~ 68 \%$ increase in the energy ratio from August to November. This means that systematic errors may still appear in terms of magnitude. Since these errors would not spatially map into a different location, there is a greater likelihood to misinterpret these as physically real gradual changes in subsurface conditions; especially, if this coincidentally corresponds to overburden compaction in a region overlying a producing reservoir unit. By incorporating the correction methodology proposed above, we mitigate such dilemmas inherent in passive monitoring.

It must, however, be noted that we assume a linear relationship exists between the psd and traveltime errors $\Delta \mathcal{T}_{i k}(\mathbf{m})$. This behavior may break down in regions where noise and Earth structure trade-off non-negligibly, e.g., in regions with strong scattering or focusing.

If this happens, we can extend the time averaging to several days or weeks and then estimate the systematic errors. By computing apparent subsurface changes as a function of the averaging interval length, we may determine the smallest possible time averaging that ensures that velocity errors do not overwhelm any potential subsurface changes that we would like to detect. This in turn would help to counteract any significant mixed or second order effects and re-enable us to linearly remove any errors.

It should also be emphasized that our method does not elucidate the physics or progeni- 
tor of these noise sources. The methodology simply images them, determines the traveltime errors produced by them, and allows the user to subtract these errors - essentially, a static error correction. The procedure thus removes these errors in the desired frequency bands, independent of whether these sources stem from a single or multiple natural - or even anthropogenic - phenomena.

\section{Extensions of the method - An outlook}

The methods and approach proposed in the previous sections constitute a step towards noise-based imaging that fully accounts for the heterogeneous and time-variable distribution of noise sources, and maximizing monitoring time resolution in a physically consistent way.

Future improvements of the method will include (i) the replacement of ray-theory kernels by finite-frequency kernels in the inversion of the ambient noise source distribution, (ii) the incorporation of frequency dependence in both the noise source and the Earth structure inversion, (iii) the implementation of this method to phase velocities for arrays with dense receiver coverage (such as SWIM), and (iv) the transition from 2D to 3D wave propagation and inversion. Ultimately, it should become possible to jointly invert for ambient noise sources and Earth structure in a fully consistent way without the need to assume equality between Green's functions and noise correlations (Hanasoge, 2013).

\section{CONCLUSIONS}

Passive seismic monitoring has many upsides over traditional 4D time-lapse surveys: It is significantly cheaper (provided that the permanent monitoring array system is already installed), does not require active sources, and can be used to provide much faster updates 
of the subsurface. In the hydrocarbon context, this cost-conscious technology would enable us to move towards the creation of real-time injection monitoring systems that could help to significantly increase production, while ensuring the integrity of both the overburden and reservoir. However, we see that the loss of repeatability due to changes in the location and strength of noise sources jeopardizes the potential applicability of noise-based monitoring.

Therefore, we develop and apply a method to quantify systematic errors in noise-based monitoring that are due to heterogeneous, non-stationary noise sources and the incorrect assumption that noise correlations equal Green's functions. Our method is based on the inversion of noise correlation asymmetries for the time-dependent distribution of noise sources, which we then use in the forward modeling of noise correlations. This allows us to determine apparent traveltime differences and the resulting apparent Earth structure.

Applying our method to real data acquired at Statoil's Oseberg field for daily passive surface wave monitoring, we find that day-to-day changes in the systematic errors would mask any actual subsurface changes that we would like to observe. Apparent velocity changes reaching $0.25 \%$ within one day are far above the average daily changes inferred from longer-term monitoring studies of hydrocarbon reservoirs (e.g. de Ridder et al., 2014; Mordret et al., 2014b).

With a synthetic inversion and an uncertainty analysis, we demonstrate that the quantified systematic traveltime errors may be used to correct traveltime measurements of a traditional ambient noise tomography where correlations are assumed to equal Green's functions. Such a correction would allow practitioners to continue using existing inversion machineries, while still accounting for heterogeneous, non-stationary noise sources. Should we not wish to correct for these artifacts, we can use this method to set a minimum thresh- 
old for which we can reliably attribute traveltime changes to actual subsurface events. This would help to minimize false alarms related to noise source non-stationarity. We thus increase the repeatability and thereby viability of time-lapse passive seismic techniques as a valuable means for monitoring subsurface conditions at increasingly higher temporal resolutions by ensuring that any significant changes imaged through our inversion algorithm are not due to fluctuations in the power spectral density of the noise sources, but rather due to structure.

\section{ACKNOWLEDGMENTS}

The authors would like to thank the editors of Geophysics, Jeffrey Shragge and Alison Malcolm, the reviewer Shravan Hanasoge, and two additional anonymous reviewers for their constructive comments and support. We would also like to thank Statoil for allowing the lead author to collaborate with its research center in Rotvoll. Additional gratitude goes to Severine Pannetier-Lescoffit and Odd Arve Solheim for handling the funding, security clearances, and hostel arrangements in Trondheim, Marianne Houbiers for her critical feedback and crash course in Seismic Unix, Andy Carter for his dialogues concerning anisotropy, Florian Wellmann for propitiously introducing him to Python merely weeks before the project's start, Frank Aavnik for his review and input, Ivar Sandø for his administrative assistance, and particularly to Eirik Aksnes for getting him up and running so hastily on the clusters.

The results here stem from a project that had to be completed within 5 months. Given this timeframe, it would have been nigh impossible to meet such deadlines without the fervent support and input of members from Statoil's Rotvoll and Oseberg Permanent Reservoir Monitoring teams and ETH Zürich's Computational Seismology group - the lead author expresses his appreciation. 
We would furthermore like to thank the operator and partners of the Oseberg license (Statoil, Petoro, Total Norge, and ConocoPhillips Skandinavia) for permission to publish this paper.

Acknowledgments for the helpful feedback provided at the 2nd TIDES (Time DEpendent Seismology) Training School - COST Action ES1401-TIDES, supported by COST (European Cooperation in Science and Technology).

This research was supported by the Swiss National Supercomputing Center (CSCS) in the form of the GeoScale and CH1 projects, by the Swiss National Science Foundation (SNF) under grant 200021_149143, and by the Netherlands Organization for Scientific Research (VIDI grant 864.11.008).

\section{APPENDIX}

\section{Electronic supplement}

The electronic supplement comes in two parts: 1) PDF and 2) Animated Clips. The PDF is broken into two sections. The first section provides background material for the animated clips. The second section delves into further detail concerning the sensitivity studies discussed in the main text. The animated clips display daily and monthly cumulative noise sensitivity kernels for four different frequency bands from April 30, 2014 to May 18, 2015. 


\section{REFERENCES}

Bakulin, A., R. Calvert, et al., 2004, Virtual source: New method for imaging and 4D below complex overburden: Presented at the 2004 SEG Annual Meeting, Society of Exploration Geophysicists.

Bensen, G., M. Ritzwoller, M. Barmin, A. Levshin, F. Lin, M. Moschetti, N. Shapiro, and Y. Yang, 2007, Processing seismic ambient noise data to obtain reliable broad-band surface wave dispersion measurements: Geophysical Journal International, 169, 1239-1260.

Bonnefoy-Claudet, S., F. Cotton, and P.-Y. Bard, 2006, The nature of noise wavefield and its applications for site effects studies: a literature review: Earth-Science Reviews, 79, $205-227$.

Boué, P., P. Poli, M. Campillo, H. Pedersen, X. Briand, and P. Roux, 2013, Teleseismic correlations of ambient noise for deep global imaging of the Earth: Geophys. J. Int., 194, $844-848$.

Brenguier, F., M. Campillo, C. Haziioannou, N. M. Shapiro, R. M. Nadeau, and E. Larose, 2008a, Postseismic relaxation along the San Andreas fault at Parkfield from continuous seismological observations: Science, 321, 1478-1481.

Brenguier, F., N. M. Shapiro, M. Campillo, V. Ferrazzini, Z. Duputel, O. Coutant, and A. Nercessian, 2008b, Towards forecasting volcanic eruptions using seismic noise: Nat. Geosci., 1, 126-130.

Bussat, S., 2015, Using ambient noise for real-time (hourly) overburden monitoring: Presented at the Third EAGE Workshop on Permanent Reservoir Monitoring 2015.

Bussat, S., and S. Kugler, 2011, Offshore ambient-noise surface-wave tomography above 0.1 $\mathrm{Hz}$ and its applications: The Leading Edge, May 2011, 514-524.

Cerjan, C., D. Kosloff, R. Kosloff, and M. Reshef, 1985, A nonreflecting boundary condition 
for discrete acoustic and elastic wave equations: Geophysics, 50, 705-708.

Claerbout, J. F., 1968, Synthesis of a layered medium from its acoustic transmission response: Geophysics, 33, 264-269.

Cupillard, P., and Y. Capdeville, 2010, On the amplitude of surface waves obtained by noise correlation and the capability to recover the attenuation: a numerical approach: Geophysical Journal International, 181, 1687-1700.

Curtis, A., P. Gerstoft, H. Sato, R. Snieder, and K. Wapenaar, 2006, Seismic interferometryturning noise into signal: The Leading Edge, 25, 1082-1092.

Dahlen, F., S.-H. Hung, and G. Nolet, 2000, Fréchet kernels for finite-frequency traveltimes - I. Theory: Geophysical Journal International, 141, 157-174.

de Ridder, S., and B. Biondi, 2013, Daily reservoir-scale subsurface monitoring using ambient seismic noise: Geophysical Research Letters, 40, 2969-2974.

de Ridder, S. A. L., and B. L. Biondi, 2015, Ambient seismic noise tomography at Ekofisk: Geophysics, 80, B167-B176.

de Ridder, S. A. L., B. L. Biondi, and R. G. Clapp, 2014, Time-lapse seismic noise correlation tomography at Valhall: Geophys. Res. Lett., 41, 6116-6122.

Duvall, T., S. Jeffferies, J. Harvey, and M. Pomerantz, 1993, Time-distance helioseismology.

Eiken, O., P. Ringrose, C. Hermanrud, B. Nazarian, T. A. Torp, and L. Høier, 2011, Lessons learned from 14 years of ccs operations: Sleipner, in salah and snøhvit: Energy Procedia, 4, $5541-5548$.

Ermert, L., A. Villaseñor, and A. Fichtner, 2016, Cross-correlation imaging of ambient noise sources: Geophysical Journal International, 204, 347-364.

Fichtner, A., 2010, Full Seismic Waveform Modelling and Inversion.: Springer, Heidelberg.

$\longrightarrow$, 2014, Source and processing effects on noise correlations: Geophys. J. Int., 197, 
$1527-1531$.

— 2015 , Source-structure trade-offs in ambient noise correlations: Geophys. J. Int., 202, 678-694.

Fichtner, A., H.-P. Bunge, and H. Igel, 2006, The adjoint method in seismology: I. theory: Physics of the Earth and Planetary Interiors, 157, 86-104.

Fichtner, A., L. Stehly, L. Ermert, and C. Boehm, 2016, Generalised interferometry - I. Theory for inter-station correlations: Geophys. J. Int., 208, 603-638.

Froment, B., M. Campillo, P. Roux, P. Gouédard, A. Verdel, and R. L. Weaver, 2010, Estimation of the effect of nonisotropically distributed energy on the apparent arrival time in correlations: Geophysics, 75, SA85-SA93.

Gimbert, F., and V. C. Tsai, 2015, Predicting short-period, wind-wave-generated seismic noise in coastal regions: Earth and Planetary Science Letters, 426, 280-292.

Groos, J. C., S. Bussat, and J. R. R. Ritter, 2012, Performance of different processing schemes in seismic noise cross-correlations: Geophysical Journal International, 188, 498512.

Halliday, D., and A. Curtis, 2008, Seismic interferometry, surface waves and source distribution: Geophysical Journal International, 175, 1067-1087.

Hanasoge, S. M., 2012, The influence of noise sources on cross-correlation amplitudes: Geophysical Journal International.

— 2013 , Measurements and kernels for source-structure inversions in noise tomography: Geophysical Journal International.

Hanasoge, S. M., and M. Branicki, 2013, Interpreting cross-correlations of one-bit filtered seismic noise: Geophysical Journal International, 195, 1811-1830.

Haned, A., E. Stutzmann, M. Schimmel, S. Kiselev, A. Davaille, and A. Yelles-Chaouche, 
2016, Gloal tomography using seismic hum: Geophys. J. Int., 204, 1222-1236.

Hillers, G., S. Husen, A. Obermann, T. Planes, E. Larose, and M. Campillo, 2015, Noisebased monitoring and imaging of aseismic transient deformation induced by the 2006 Basel reservoir stimulation: Geophysics, 80, KS51-KS68.

Kimman, W., and J. Trampert, 2010, Approximations in seismic interferometry and their effects on surface waves: Geophysical Journal International, 182, 461-476.

Larose, E., L. Margerin, A. Derode, B. van Tiggelen, M. Campillo, N. Shapiro, A. Paul, L. Stehly, and M. Tanter, 2006, Correlation of random wavefields: An interdisciplinary review: Geophysics, 71, SI11-SI21.

Latorre, D., A. Amato, M. Cattaneo, S. Carannante, and A. Michelini, 2014, Man-induced low-frequency seismic events in italy: Geophysical Research Letters, 41, 8261-8268.

Lawrence, J. F., M. Denolle, K. J. Seats, and G. A. Prieto, 2013, A numeric evaluation of attenuation from ambient noise correlation functions: Journal of Geophysical Research, 118, 1-12.

Lawrence, J. F., and G. A. Prieto, 2011, Attenuation tomography of the western United States from ambient seismic noise: Journal of Geophysical Research, 116, 1978-2012.

Lin, F.-C., M. P. Moschetti, and M. H. Ritzwoller, 2008, Surface wave tomography of the western United States from ambient noise: Rayleigh and Love wave phase velocity maps: Geophys. J. Int., 173, 281-298.

Lobkis, O. I., and R. L. Weaver, 2001, On the emergence of the greens function in the correlations of a diffuse field: The Journal of the Acoustical Society of America, 110, $3011-3017$.

Luo, Y., and G. T. Schuster, 1991, Wave-equation traveltime inversion: Geophysics, 56, 645-653. 
Malcolm, A. E., J. A. Scales, and B. A. van Tiggelen, 2004, Extracting the green function from diffuse, equipartitioned waves: Physical Review E, 70, 015601.

Malcolm, A. E., and J. Trampert, 2011, Tomographic errors from wave front healing: more than just a fast bias: Geophysical Journal International, 185, 385-402.

Meier, U., N. M. Shapiro, and F. Brenguier, 2010, Detecting seasonal variations in seismic velocities within the Los Angeles basin from correlations of ambient seismic noise: Geophys. J. Int., 181, 985-996.

Montelli, R., G. Nolet, F. Dahlen, G. Masters, E. R. Engdahl, and S.-H. Hung, 2004, Finite-frequency tomography reveals a variety of plumes in the mantle: Science, 303, $338-343$.

Mordret, A., M. Landès, N. Shapiro, S. Singh, and P. Roux, 2014a, Ambient noise surface wave tomography to determine the shallow shear velocity structure at valhall: depth inversion with a neighbourhood algorithm: Geophysical Journal International, 198, 15141525.

Mordret, A., N. Shapiro, and S. Singh, 2014b, Seismic noise-based time-lapse monitoring of the Valhall overburden: Geophys. Res. Lett., 41, 49454952.

Mulgaria, F., 2012, The seismic noise wavefield is not diffuse: Journal of the Acoustical Society of America, 131, doi: 10.1121/1.3689551.

Nakata, N., J. P. Chang, J. F. Lawrence, and P. Boué, 2015, Body wave extraction and tomography at Long Beach, California, with ambient-noise interferometry: J. Geophys. Res., 120, 1159-1173.

Nishida, K., 2014, Source spectra of seismic hum: Geophysical Journal International, 199, $416-429$.

Nishida, K., and J.-P. Montagner, 2009, Global surface wave tomography using seismic 
hum: Science, 326, 5949 .

Obermann, A., B. Froment, M. Campillo, E. Larose, T. Planes, B. Valette, J.-H. Chen, and Q. Y. Liu, 2014, Seismic noise correlations to image structural and mechanical changes associated with the Mw 7.9 2008 Wenchuan earthquake: J. Geophys. Res., 119, doi:10.1002/2013JB010932.

Obermann, A., T. Kraft, E. Larose, and S. Wiemer, 2015, Potential of ambient seismic noise techniques to monitor the St. Gallen geothermal site (Switzerland): J. Geophys. Res., 120, doi:10.1002/2014JB011817.

Obermann, A., T.Planes, E. Larose, and M. Campillo, 2013, Imaging preeruptive and coeruptive structural and mechanical changes of a volcano with ambient seismic noise: J. Geophys. Res., 118, 1-10.

Peterson, J., 1993, Observations and modeling of seismic background noise.

Plessix, R.-E., 2006, A review of the adjoint-state method for computing the gradient of a functional with geophysical applications: Geophysical Journal International, 167, 495503.

Raknes, E. B., W. Weibull, and B. Arntsen, 2015, Seismic imaging of the carbon dioxide gas cloud at sleipner using 3d elastic time-lapse full waveform inversion: International Journal of Greenhouse Gas Control, 42, 26-45.

Rickett, J., and J. Claerbout, 1999, Acoustic daylight imaging via spectral factorization: Helioseismology and reservoir monitoring: The leading edge, 18, 957-960.

Ruigrok, E., D. Draganov, and K. Wapenaar, 2008, Global-scale seismic interferometry: theory and numerical examples: Geophysical Prospecting, 56, 395-417.

Sabra, K. G., P. Gerstoft, P. Roux, and W. A. Kuperman, 2005, Surface wave tomography from microseisms in Southern California: Geophys. Res. Lett., 32, 
doi:10.1029/2005GL023155.

Schimmel, M., 1999, Phase cross-correlations: Design, comparisons, and applications: Bulletin of the Seismological Society of America, 89, 1366-1378.

Schimmel, M., E. Stutzmann, and J. Gallart, 2011, Using instantaneous phase coherence for signal extraction from ambient noise data at a local to a global scale: Geophys. J. Int., 184, 494-506.

Schulte-Pelkum, V., P. S. Earle, and F. L. Vernon, 2004, Strong directivity of oceangenerated seismic noise: Geochemistry, Geophysics, Geosystems, 5.

Seats, K. J., J. F. Lawrence, and G. A. Prieto, 2012, Improved ambient noise correlation functions using welch s method: Geophysical Journal International, 188, 513-523.

Sens-Schoenfelder, C., R. Snieder, and S. Staehler, 2015, The lack of equipartitioning n global body wave coda: Geophysical Research Letters, 42, 7483-7489.

Shapiro, N. M., M. Campillo, L. Stehly, and M. H. Ritzwoller, 2005, High-resolution surfacewave tomography from ambient seismic noise: Science, 307, 1615-1618.

Snieder, R., 2004, Extracting the greens function from the correlation of coda waves: A derivation based on stationary phase: Physical Review E, 69, 046610.

Stehly, L., M. Campillo, and N. Shapiro, 2006, A study of the seismic noise from its longrange correlation properties: Journal of Geophysical Research: Solid Earth (1978-2012), 111.

Stutzmann, E., F. Ardhuin, M. Schimmel, A. Mangeney, and G. Patau, 2012, Modelling long-term seismic noise in various environments: Geophys. J. Int., 191, 707-722.

Tanimoto, T., S. Ishimaru, and C. Alvizuri, 2006, Seasonality in particle motion of microseisms: Geophysical Journal International, 166, 253-266.

Tarantola, A., 1988, Theoretical background for the inversion of seismic waveforms including 
elasticity and attenuation: Pure and Applied Geophysics, 128, 365-399.

Tromp, J., Y. Luo, S. Hanasoge, and D. Peter, 2010, Noise cross-correlation sensitivity kernels: Geophysical Journal International, 183, 791-819.

Tsai, V. C., 2009, On establishing the accuracy of noise tomography travel-time measurements in a realistic medium: Geophysical Journal International, 178, 1555-1564.

— 2011 , Understanding the amplitudes of noise correlation measurements: Journal of Geophysical Research: Solid Earth (1978-2012), 116.

Verbeke, J., L. Boschi, L. Stehly, E. Kissling, and A. Michelini, 2012, High-resolution Rayleigh-wave velocity maps of central Europe from a dense ambient-noise data set: Geophys. J. Int., 188, 1173-1187.

Verdon, J., J.-M. Kendall, D. White, and D. Angus, 2011, Linking microseismic event observations with geomechanical models to minimise the risks of storing co 2 in geological formations: Earth and Planetary Science Letters, 305, 143-152.

Virieux, J., 1984, Sh-wave propagation in heterogeneous media: velocity-stress finitedifference method: Geophysics, 49, 1933-1942.

Wapenaar, K., D. Draganov, R. Snieder, X. Campman, and A. Verdel, 2010, Tutorial on seismic interferometry: Part 1basic principles and applications: Geophysics, 75, 75A19575A209.

Wapenaar, K., and J. Fokkema, 2006, Greens function representations for seismic interferometry: Geophysics, 71, SI33-SI46.

Wapenaar, K., E. Slob, and R. Snieder, 2006, Unified greens function retrieval by cross correlation: Physical Review Letters, 97, 234301.

Weaver, R. L., and O. I. Lobkis, 2001, Ultrasonics without a source: Thermal fluctuation correlations at mhz frequencies: Physical Review Letters, 87, 134301. 
— 2004 , Diffuse fields in open systems and the emergence of the greens function (1): The Journal of the Acoustical Society of America, 116, 2731-2734.

Wielandt, E., 1987, On the validity of the ray approximation for interpreting delay times, in Seismic tomography: Springer, 85-98.

Woodard, M., 1997, Implications of localized, acoustic absorption for heliotomographic analysis of sunspots: The Astrophysical Journal, 485, 890.

Zhan, Z., V. C. Tsai, and R. W. Clayton, 2013, Spurious velocity changes caused by temporal variations in ambient noise frequency content: Geophys. J. Int., 194, 1574-1581. 


\section{LIST OF FIGURES}

$1 \quad$ SWIM array layout with its location given in the inset. Receiver spacing is $\sim 50$ $\mathrm{m}$ for the array's outer border and decreases to $\sim 25 \mathrm{~m}$ for the inner section. The array's outer right-side's offset (from receiver 137 to 172 ) is $1737.2 \mathrm{~m}$ and the horizontal separation of the right-side's inner and outer segments roughly halfway down the array (from receiver 96 to 155$)$ is $298.4 \mathrm{~m}$. Due to the array's symmetry, similar properties apply to its left-side.

2 Correlation functions for July 21 and July 22 for selected receiver-receiver azimuths in the frequency band 1.15-1.55 Hz. The logarithmic causal-to-acausal signal energy ratio, explained in more detail in the following section, is given to the right of the plotted correlations. For a homogeneous noise source distribution, the logarithmic energy ratios would be equal to zero. Instead, they vary significantly between -2.64 and 1.88 , with dependence on both azimuth and time. This indicates a pronounced spatio-temporal variability of the noise sources that can be observed robustly. For inter-station pair 134-162 (black), its az-

imuth points slightly west of north, as indicated by the azimuth compass. Receiver 134 thus lies at the nock, whereas receiver 162 is at the arrow tip - in terms of the receivers' relative orientation to each other, and not absolute position in the array. More energy comes from the north than from the south for this receiver pair on July 22 (i.e., causal amplitudes are larger than acausal). This is also supported by receiver pair 21-130, where more energy arrives from a northwesterly direction than from the southeast on this same day (i.e., acausal amplitudes are larger than causal with this pair's orientation flipped to that of pair 134-162). The fact that we observe spurious arrivals of non-zero amplitude information and an elongated wave train for receiver pair 16-111 on July 22 indicates that noise sources are moving throughout the day and that even shorter time intervals would be necessary to better constrain their azimuthal position. 
3 Correlation function for receiver pair 36-145. Black dashed lines mark the measurement windows $w_{-}(t)$ and $w_{+}(t)$ from equation (4), centered around the acausal and causal Scholte waves, respectively. Gray dashed lines indicate windows used to estimate signal-to-noise $(\mathrm{s} / \mathrm{n})$ ratios, needed to eliminate correlation functions of insufficient quality. These windows are centered at lag times where no direct arrivals are expected. In this example, the amplitude ratio is 0.55 , and the $\mathrm{s} / \mathrm{n}$ is 234.4 and 464.1 on the causal and acausal branch, respectively. The s/n ratio is defined as $E_{+} / \int\left[w_{n+}(t) \bar{C}(t)\right]^{2} d t$ for the causal side and $E_{-}(t) / \int\left[w_{n-}(t) \bar{C}(t)\right]^{2} d t$ for the acausal, where $w_{n+}(t)$ and $w_{n-}(t)$ respectively denote causal and acausal windows lacking any expected meaningful signal.

4 Sensitivity kernels for the noise source psd for receiver pair 36-145, as indicated by the black triangles. Plotted in the background is the annulus of the azimuthally homogeneous noise source psd used as the initial model in the noise source inversion. (a) Finite-frequency kernel containing the first and higher Fresnel zones. Darker areas indicate regions where the $p s d$ (within the annulus) should be increased, and vice versa. (b) Ray-theoretical simplification of the finite-frequency kernel (Ermert et al., 2016) used to constrain noise sources with low computational costs on a daily basis.

5 Binning map highlighting the azimuthal coverage for all receiver pairs used for July 21 (top) and July 22 (bottom), 2014, in the frequency band 1.15-1.55 Hz. The bin hits run along great circle segments extending $1000 \mathrm{~km}$ radially from the center of every interstation pair used. Latitudes range from $50.6^{\circ} \mathrm{N}$ to $70.6^{\circ} \mathrm{N}$ and longitudinally from $17.2^{\circ} \mathrm{W}$ to $22.8^{\circ} \mathrm{E}$, with a resolution of $0.1^{\circ}$ in both directions. Though July 22 uses 90 pairs less than July 21, we notice only negligible differences in azimuthal fold coverage between these two days. The differences that we observe in Figure 6 are thus not due to a biased data selection, but are genuinely driven by the non-stationary noise sources. 
6 Cumulative noise source kernels for all receiver pairs for July 21 (top) and July 22 (bottom), 2014, in the frequency band 1.15-1.55 Hz. Being directions of steepest ascent, the kernels provide a first image of the noise source $p s d$. Within the azimuthal range marked by green tones, the $p s d$ should increase relative to the azimuthally homogeneous initial $p s d$. Within the azimuthal range marked by red tones, the $p s d$ should decrease.

$7 \quad$ Noise source $p s d$ for July 21 and July 22, 2014. The annulus with non-zero noise source strength is centered around the SWIM array, marked in red.

8 Traveltime differences $\Delta \mathcal{T}_{i k}(\mathbf{m})$ between synthetic correlations $\bar{C}\left(\mathbf{x}_{i}, \mathbf{x}_{k}, t\right)$ computed for an isotropic noise source distribution and artificial data $\bar{C}^{\mathrm{o}}\left(\mathbf{x}_{i}, \mathbf{x}_{k}, t\right)$ computed for the noise source distributions on July 21 and July 22, 2014, shown in Figures 6 and 7. We observe that the biases show strong anisotropy, a direct result of the anisotropic $p s d$.

$9 \quad$ Apparent group velocity variations $\delta v$ on July 21 and July 22, 2014, induced by the heterogeneous distribution of noise sources.

10 Apparent fractional changes of the group velocity variations from July 21 to July 22, 2014, induced by temporal changes in the noise sources, shown in Figures 6 and 7 . The reference velocity is $300 \mathrm{~m} / \mathrm{s}$. Without knowing that these are systematic errors due to assuming correlation and Green's function equivalency, we may wrongly attribute these to localized subsurface perturbations.

11 Finite-frequency inversion results attempting to image the true structure perturbation rendered in a). b) Baseline case I, where the non-stationary noise sources are taken into account and assumed to be known perfectly. This is the best possible reconstruction using the given data. c) Case II where we image time-lapse velocity perturbations assuming that noise sources are homogeneous and not variable in time. d) Case III where we do correct for noise source non-stationarity biases by imaging the noise source $p s d$. Correcting 
for noise source biases allows us to remove spurious artifacts and better identify the true structural change. In case II, it is difficult to argue whether the imaged positive anomaly in the northwestern corner is genuine. With the correction applied in case III, we see that this anomaly is merely an artifact.

12 Traveltime differences between synthetic correlations $\bar{C}\left(\mathbf{x}_{i}, \mathbf{x}_{k}, t\right)$ computed for an isotropic noise source distribution and artificial data $\bar{C}^{\mathrm{o}}\left(\mathbf{x}_{i}, \mathbf{x}_{k}, t\right)$ computed for the noise source distributions on July 21 (blue) and July 22 (red), shown in Figures 6 and 7 - now plotted against inter-station pair distance. Systematic errors depend on psd azimuths with respect to inter-station pair azimuths and are less sensitive to inter-station pair distance.

13 (a) Weighted angular mean of the noise source azimuth as a function of daily averaging (solid line) and of monthly averaging (dotted line) for frequency bands 0.55-0.95 $\mathrm{Hz}$ (green) and 1.15-1.55 Hz (magenta). (b) Mean absolute energy ratio of correlations at corresponding angular mean. As we invert for noise source psd by using energy ratios, we can use the energy ratios as a $p s d$ analogue. We observe that noise source non-stationarity is spatially, frequency, and temporally dependent and that temporal dependence can be reduced by averaging over longer time periods. Furthermore, we can infer on which days we are more likely to produce false alarms or fail to correctly identify structural changes, should we decide to not set a minimum threshold or remove artificial changes 


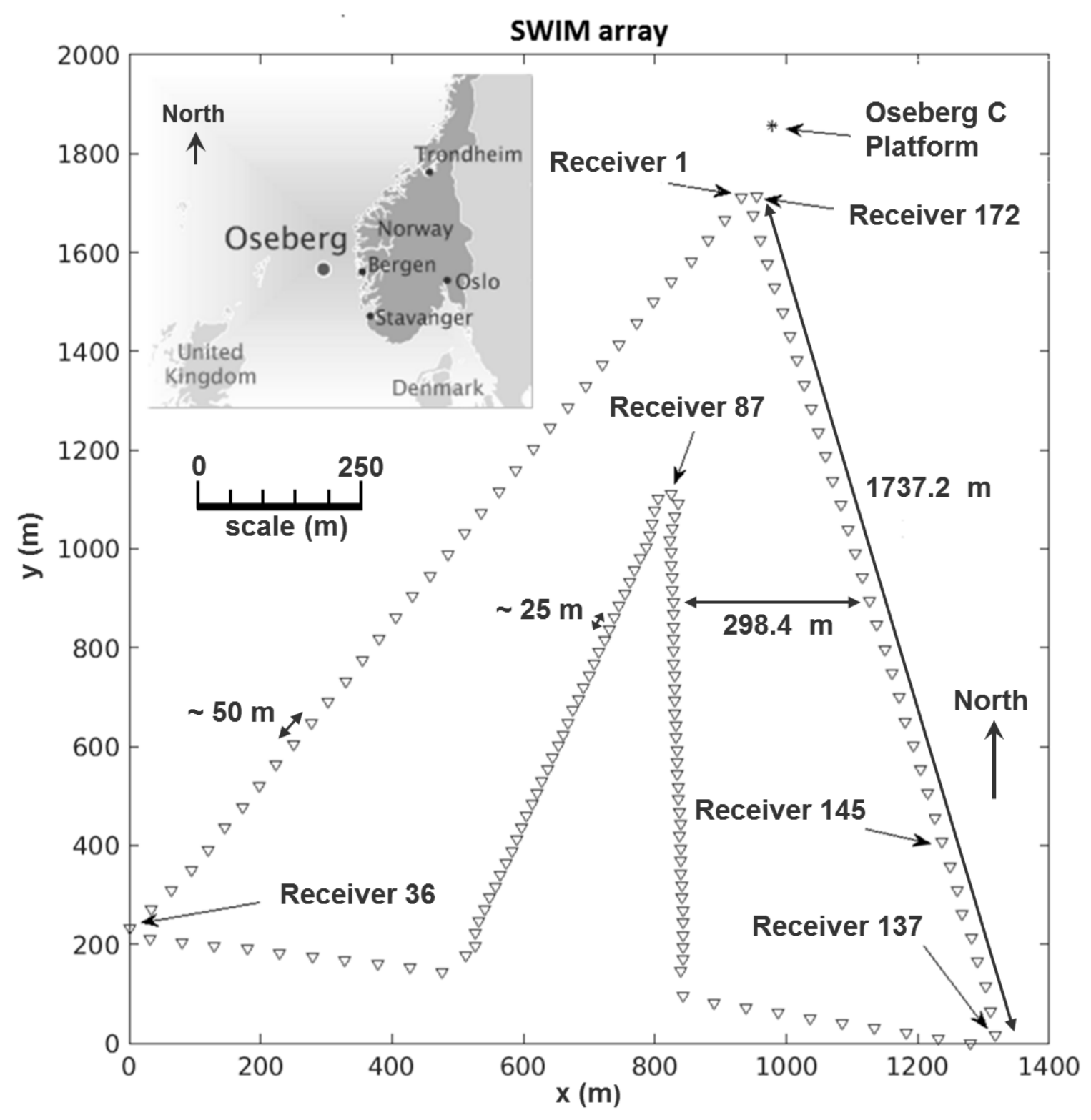

Figure 1: SWIM array layout with its location given in the inset. Receiver spacing is $\sim 50$ $\mathrm{m}$ for the array's outer border and decreases to $\sim 25 \mathrm{~m}$ for the inner section. The array's outer right-side's offset (from receiver 137 to 172 ) is $1737.2 \mathrm{~m}$ and the horizontal separation of the right-side's inner and outer segments roughly halfway down the array (from receiver 96 to 155$)$ is $298.4 \mathrm{~m}$. Due to the array's symmetry, similar properties apply to its left-side. 


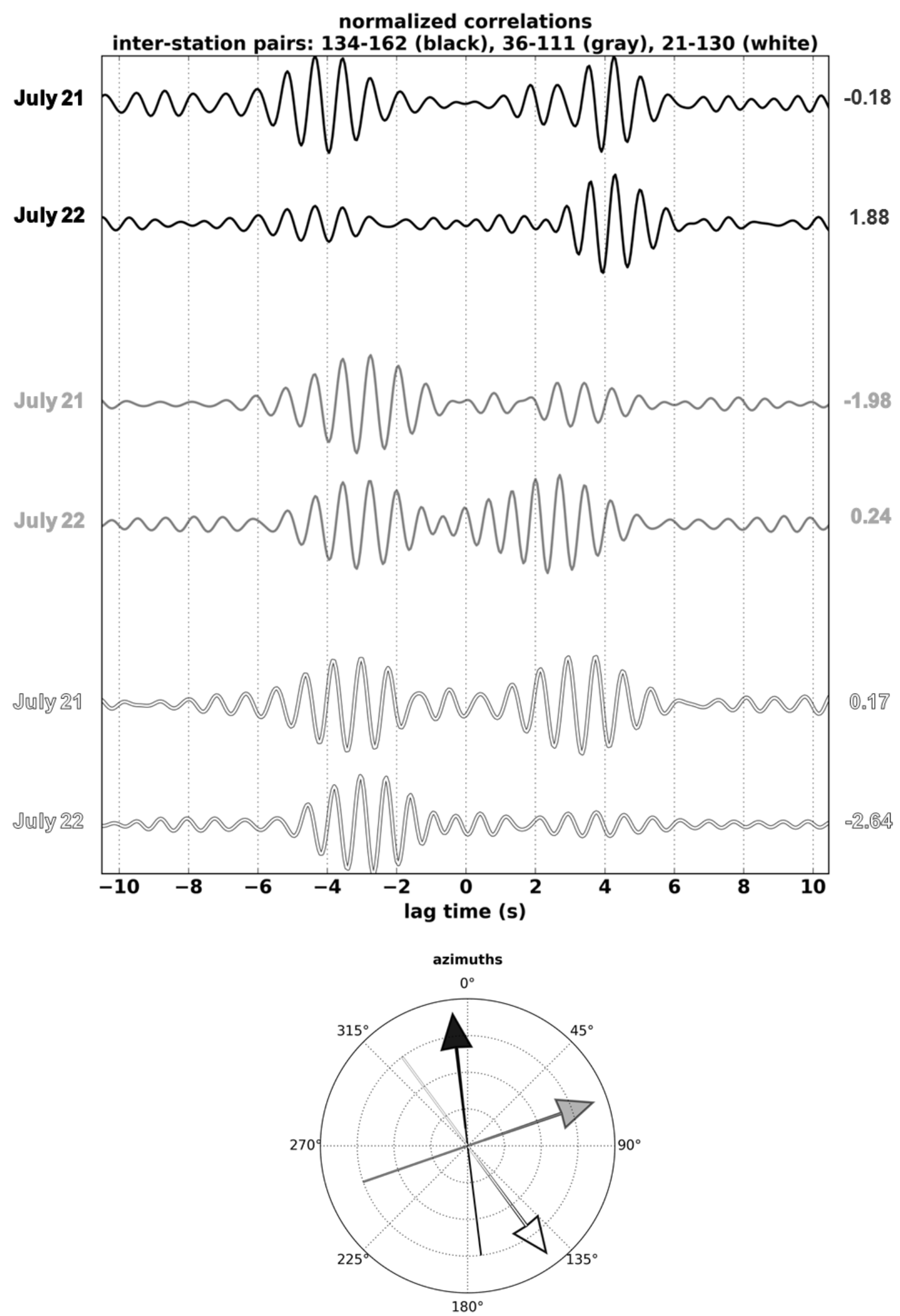

Figure 2: Correlation functions for July 21 and July 22 for selected receiver-receiver az- 


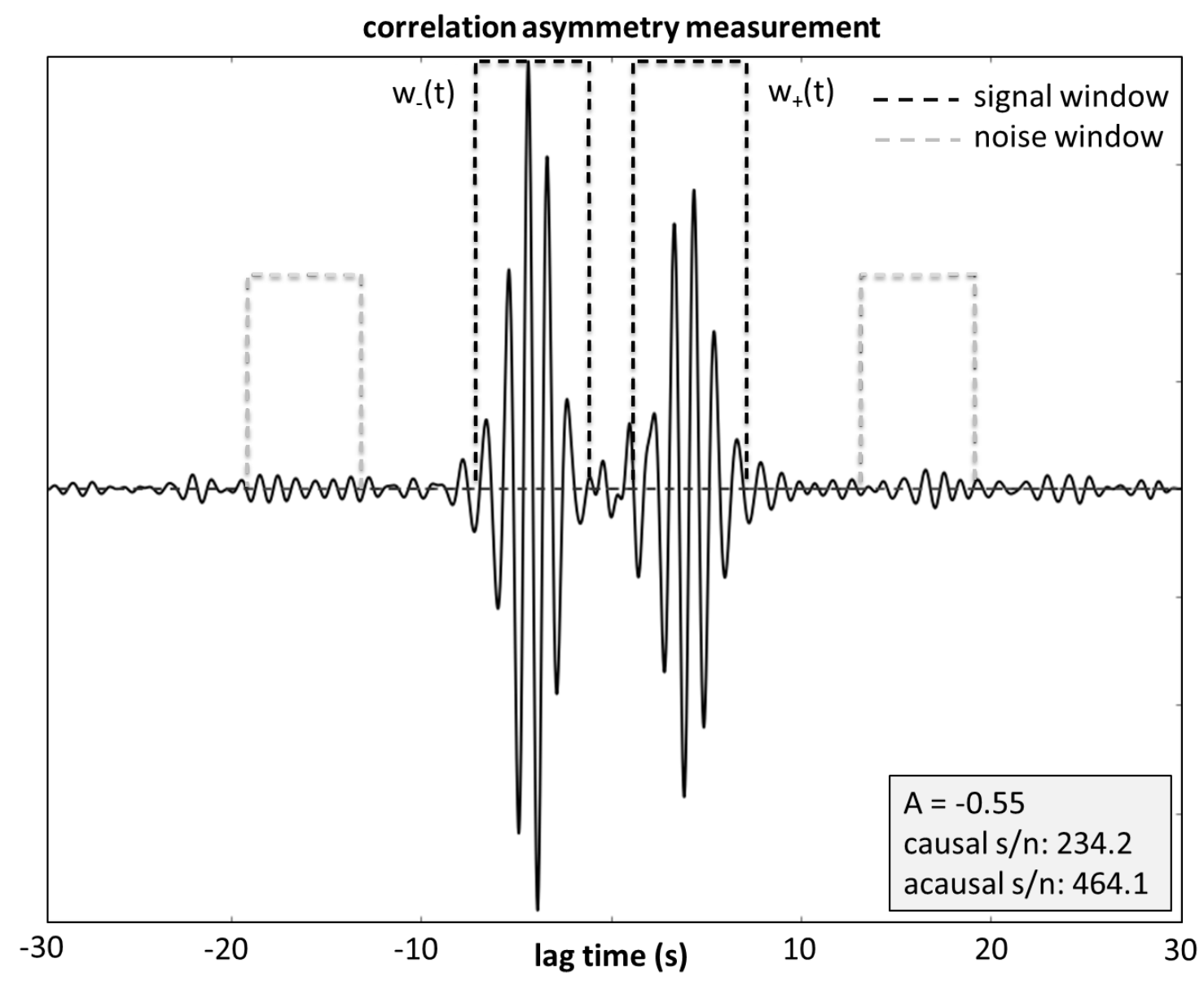

Figure 3: Correlation function for receiver pair 36-145. Black dashed lines mark the measurement windows $w_{-}(t)$ and $w_{+}(t)$ from equation (4), centered around the acausal and causal Scholte waves, respectively. Gray dashed lines indicate windows used to estimate signal-to-noise $(\mathrm{s} / \mathrm{n})$ ratios, needed to eliminate correlation functions of insufficient quality. These windows are centered at lag times where no direct arrivals are expected. In this example, the amplitude ratio is 0.55 , and the $\mathrm{s} / \mathrm{n}$ is 234.4 and 464.1 on the causal and acausal branch, respectively. The s/n ratio is defined as $E_{+} / \int\left[w_{n+}(t) \bar{C}(t)\right]^{2} d t$ for the causal side and $E_{-}(t) / \int\left[w_{n-}(t) \bar{C}(t)\right]^{2} d t$ for the acausal, where $w_{n+}(t)$ and $w_{n-}(t)$ respectively denote causal and acausal windows lacking any expected meaningful signal. 


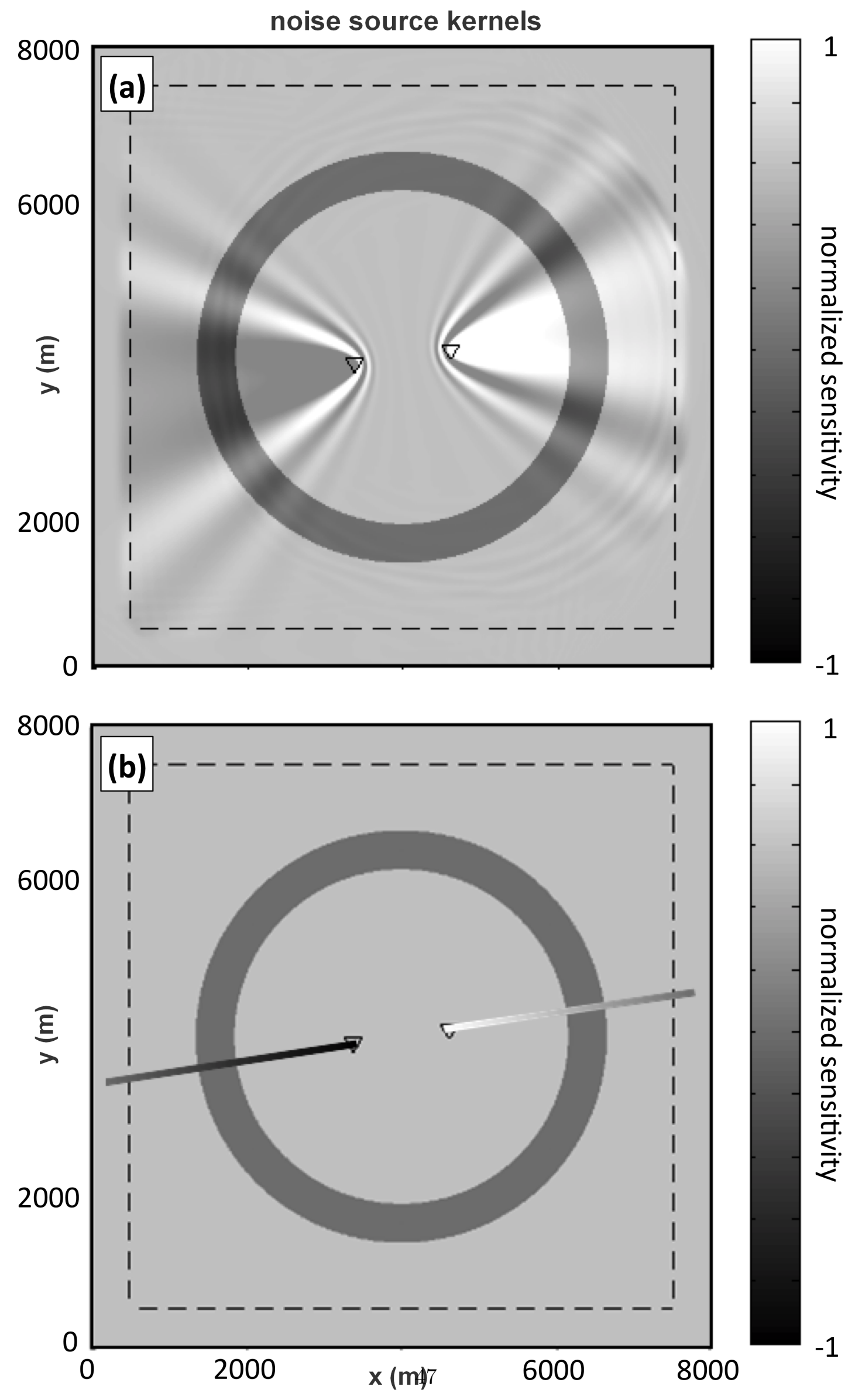



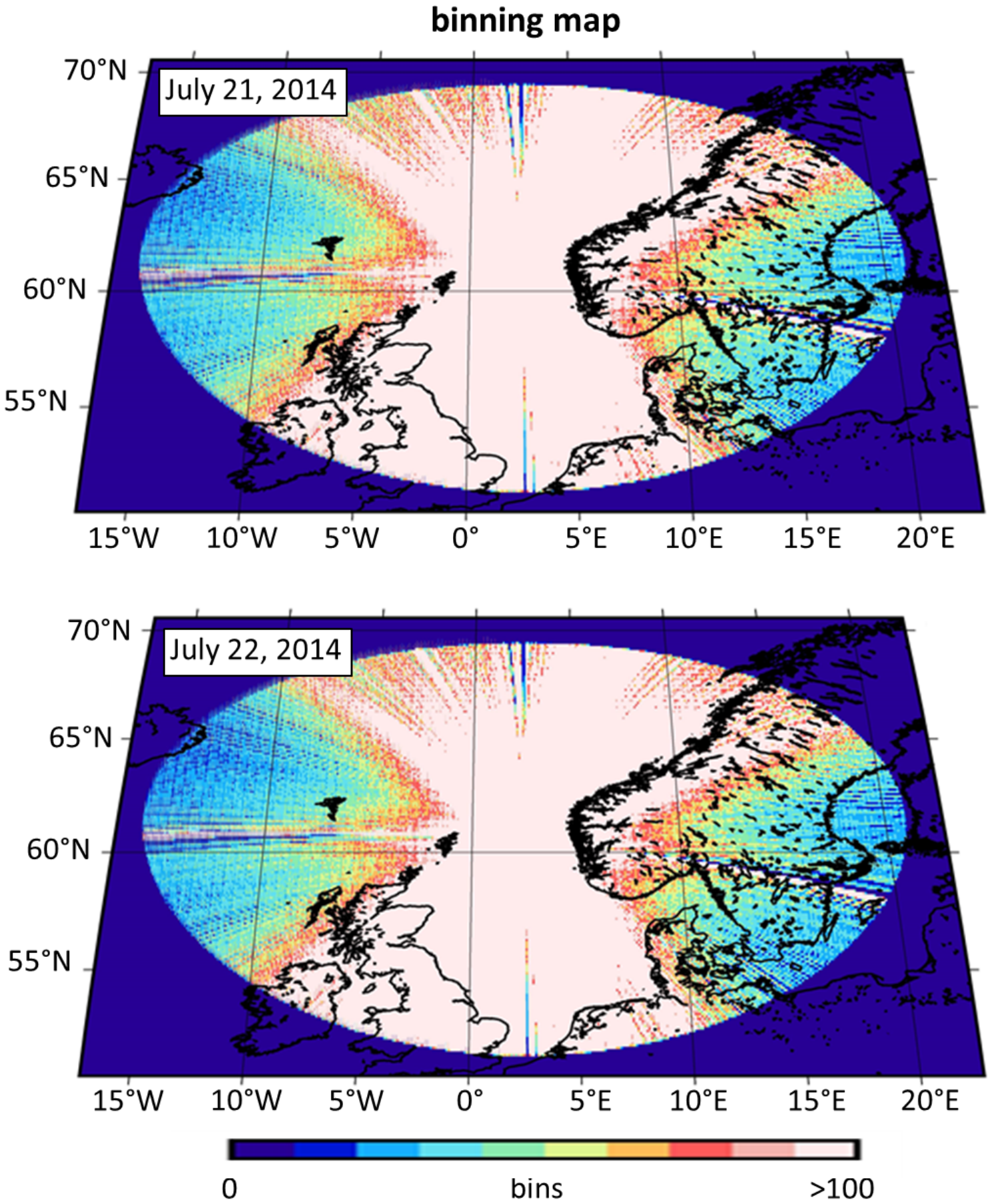

Figure 5: Binning map highlighting the azimuthal coverage for all receiver pairs used for July 21 (top) and July 22 (bottom), 2014, in the frequency band 1.15-1.55 Hz. The bin hits run along great circle segments extending $1000 \mathrm{~km}$ radially from the center of every interstation pair used. Latitudes range from $50.6_{48}^{\circ} \mathrm{N}$ to $70.6^{\circ} \mathrm{N}$ and longitudinally from $17.2^{\circ} \mathrm{W}$ to $22.8^{\circ} \mathrm{E}$, with a resolution of $0.1^{\circ}$ in both directions. Though July 22 uses 90 pairs less 

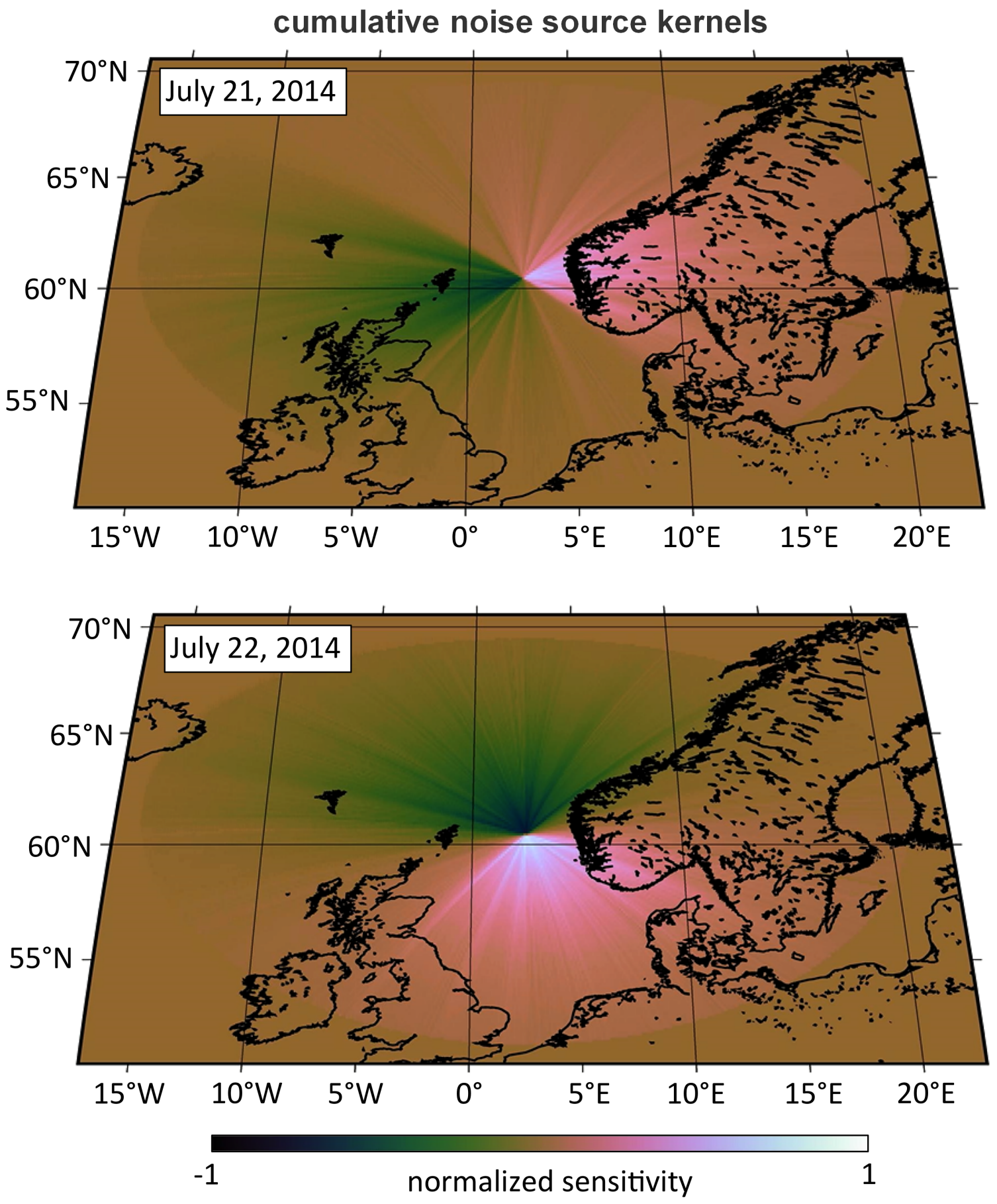

Figure 6: Cumulative noise source kernels for all receiver pairs for July 21 (top) and July 22 (bottom), 2014, in the frequency band 1.15-1.55 Hz. Being directions of steepest ascent, the kernels provide a first image of the noise source $p s d$. Within the azimuthal range marked by green tones, the $p s d$ should increase relative to the azimuthally homogeneous initial $p s d$. Within the azimuthal range marked by red tones, the $p s d$ should decrease. 


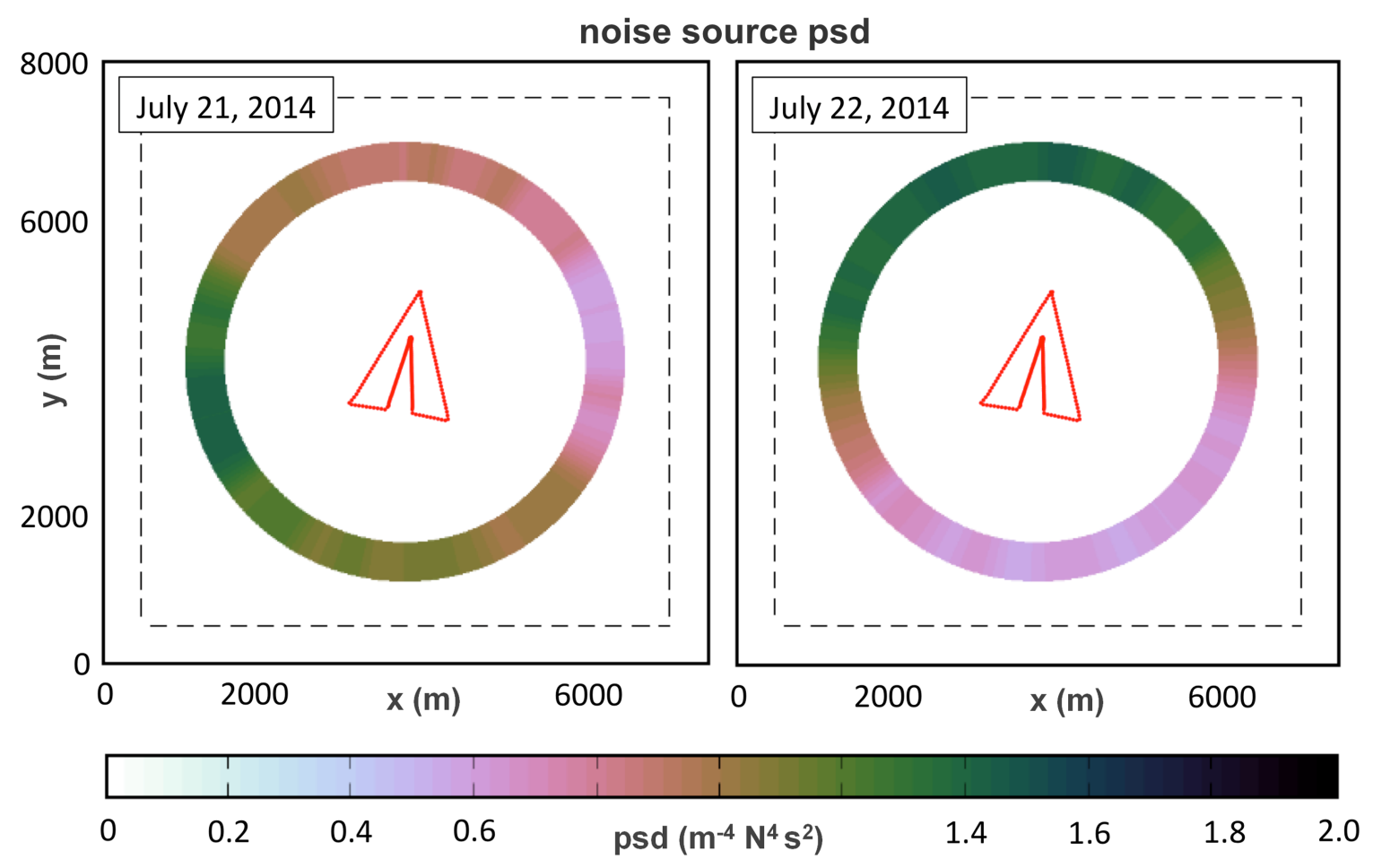

Figure 7: Noise source psd for July 21 and July 22, 2014. The annulus with non-zero noise source strength is centered around the SWIM array, marked in red. 


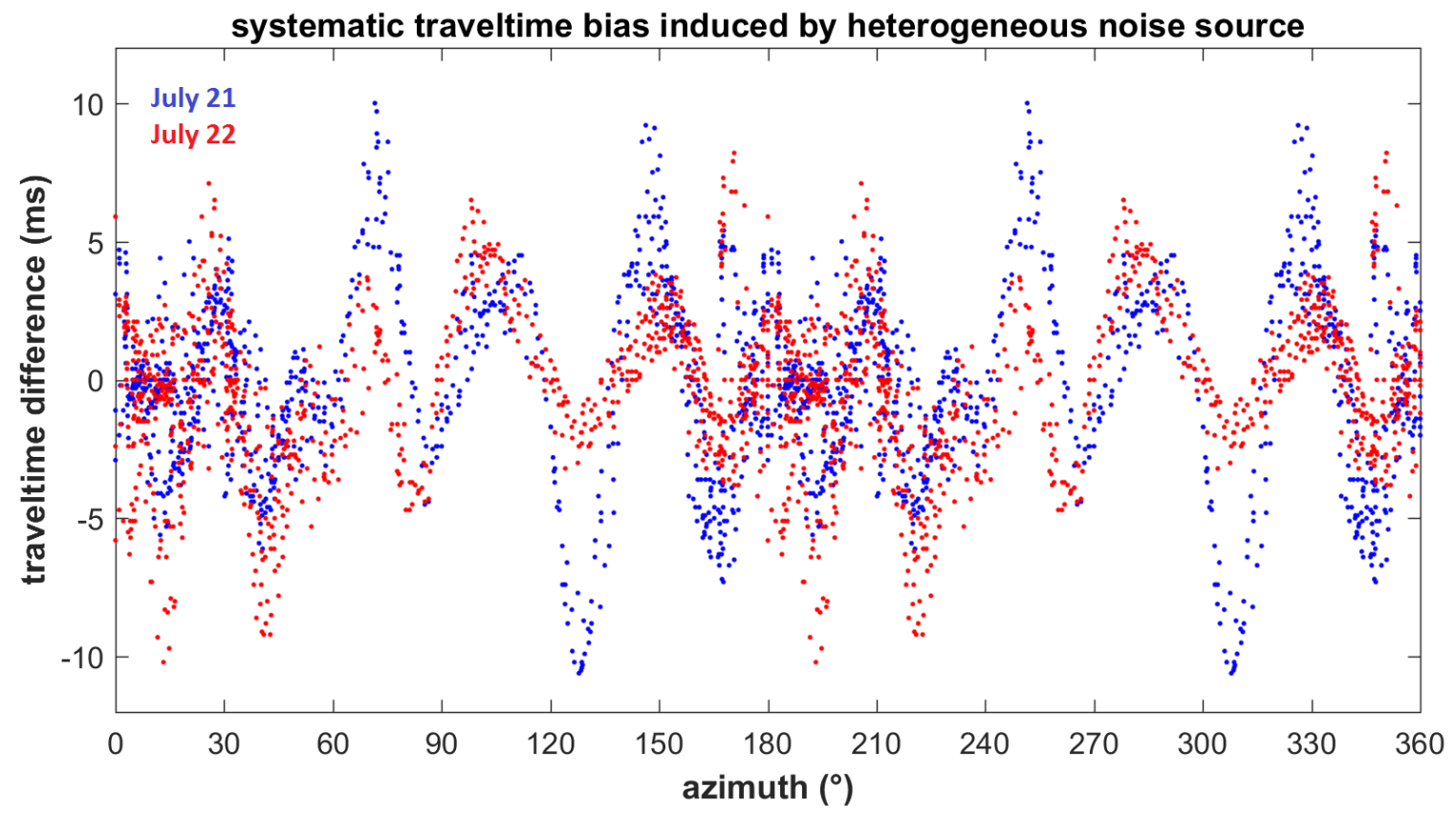

Figure 8: Traveltime differences $\Delta \mathcal{T}_{i k}(\mathbf{m})$ between synthetic correlations $\bar{C}\left(\mathbf{x}_{i}, \mathbf{x}_{k}, t\right)$ computed for an isotropic noise source distribution and artificial data $\bar{C}^{\mathrm{o}}\left(\mathbf{x}_{i}, \mathbf{x}_{k}, t\right)$ computed for the noise source distributions on July 21 and July 22, 2014, shown in Figures 6 and 7. We observe that the biases show strong anisotropy, a direct result of the anisotropic $p s d$. 

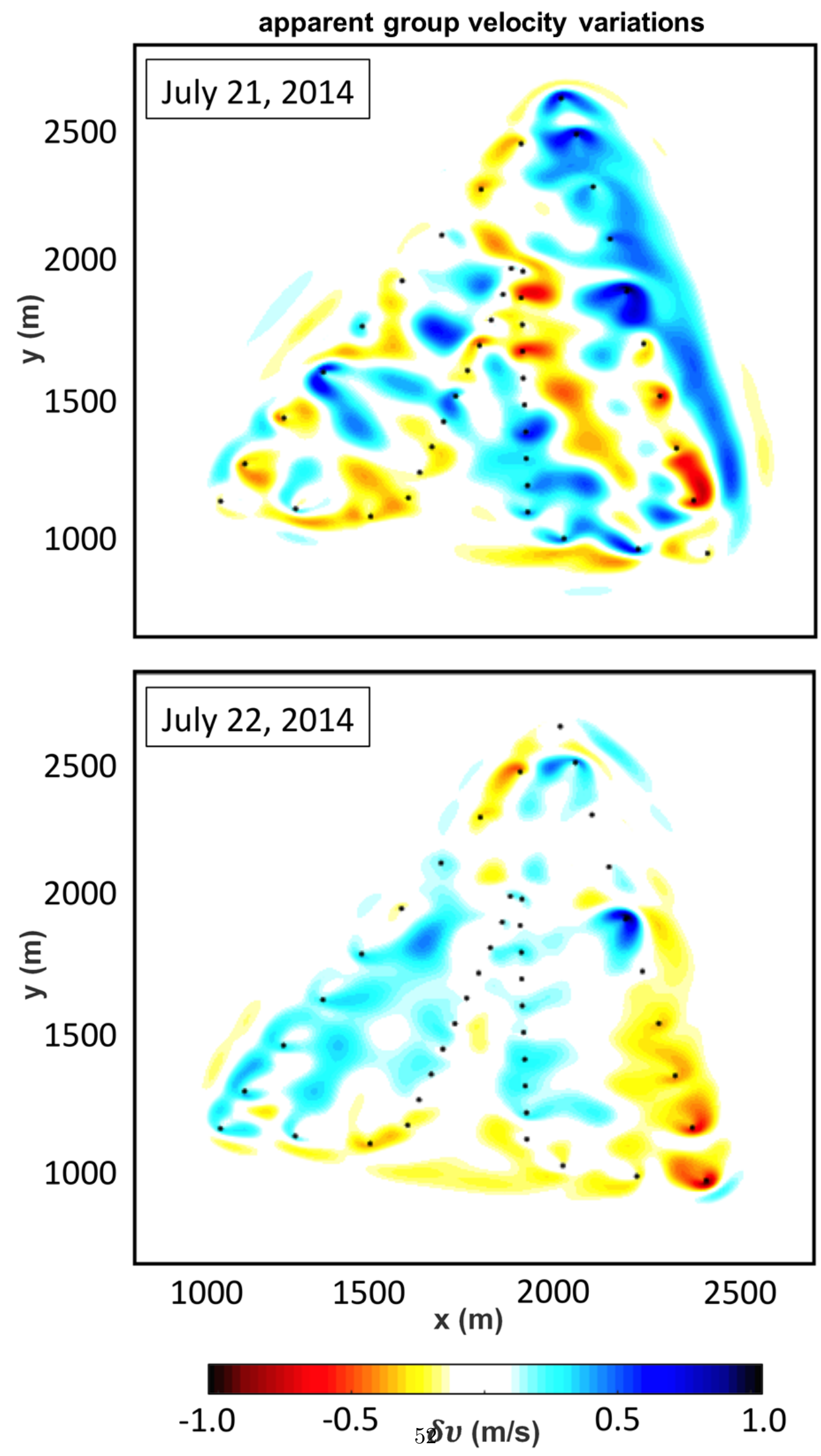


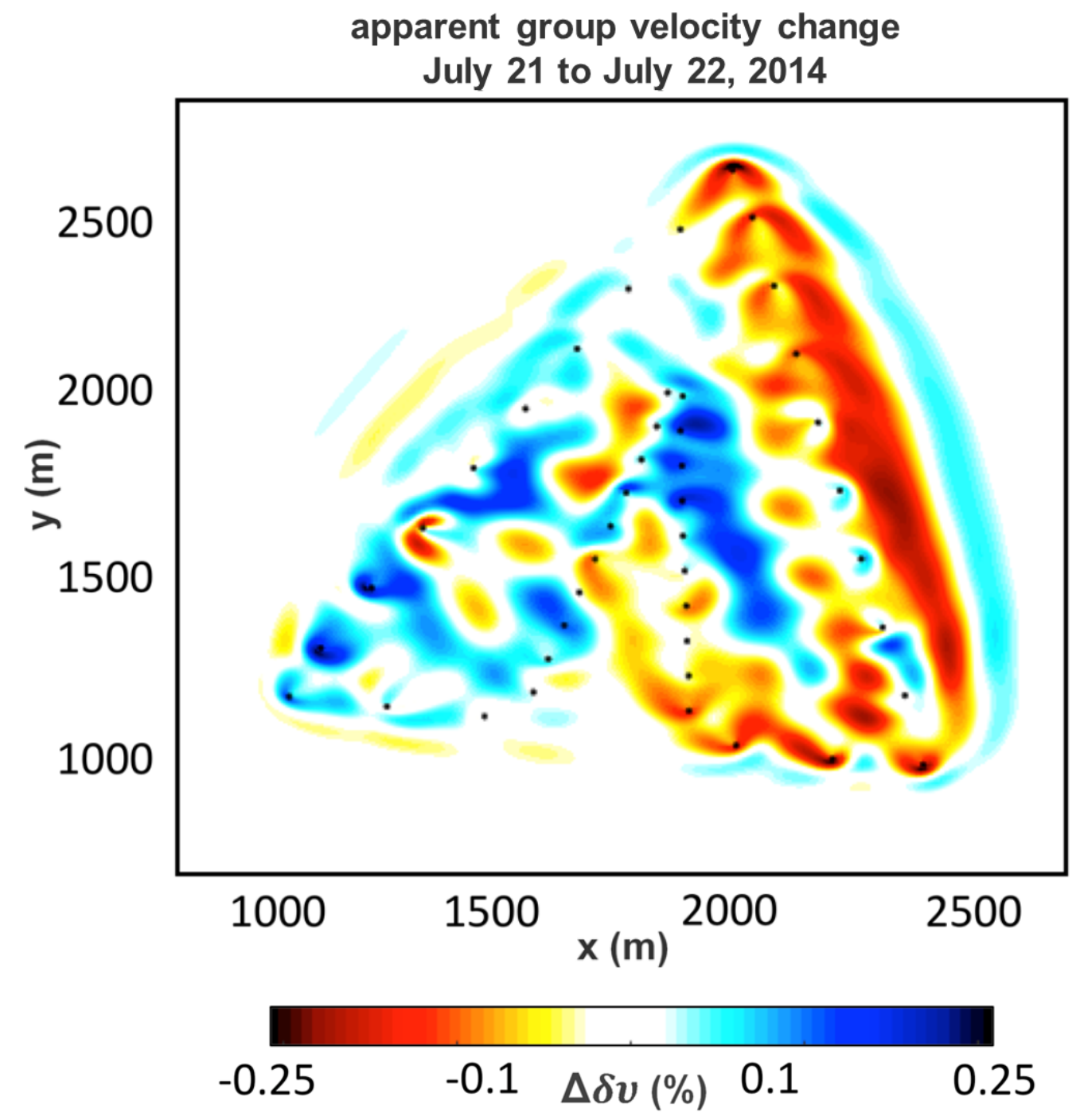

Figure 10: Apparent fractional changes of the group velocity variations from July 21 to July 22, 2014, induced by temporal changes in the noise sources, shown in Figures 6 and 7 . The reference velocity is $300 \mathrm{~m} / \mathrm{s}$. Without knowing that these are systematic errors due to assuming correlation and Green's function equivalency, we may wrongly attribute these to localized subsurface perturbations. 
(a) Target velocity perturbation

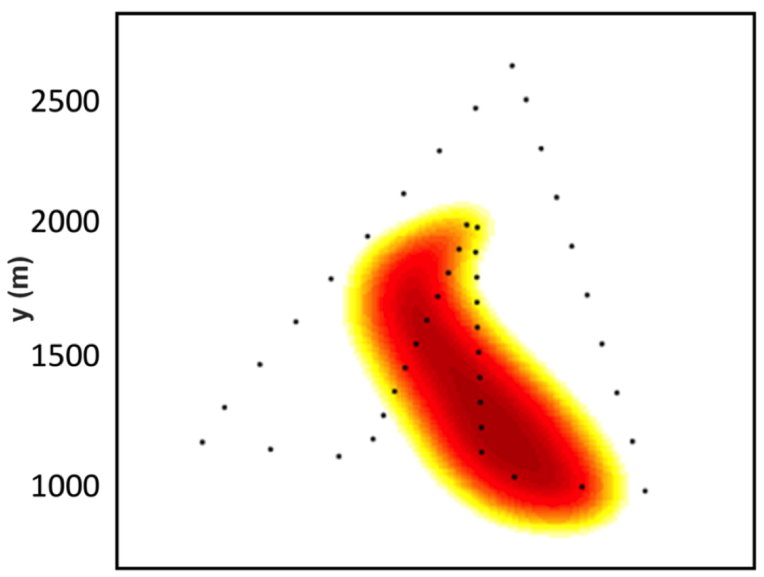

(c) Ignoring non-stationary $p s d$.

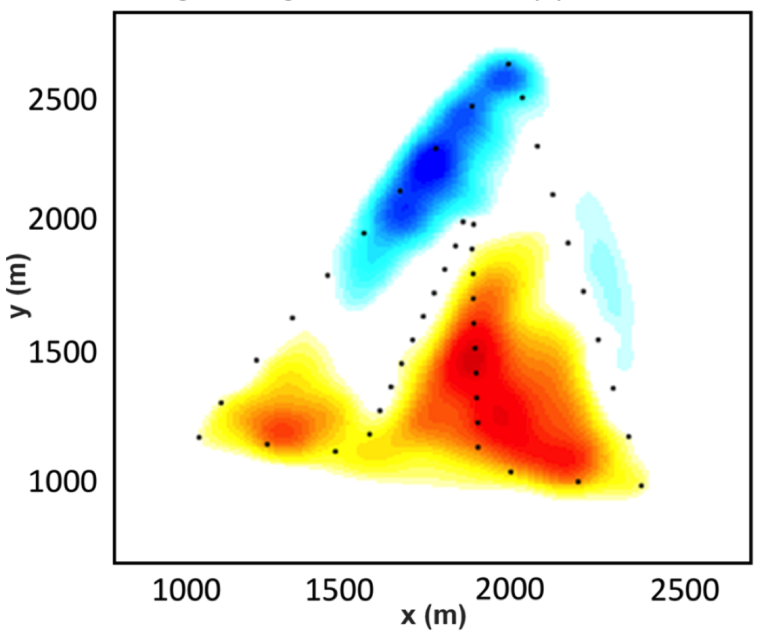

(b) Baseline. Perfectly known noise psd.

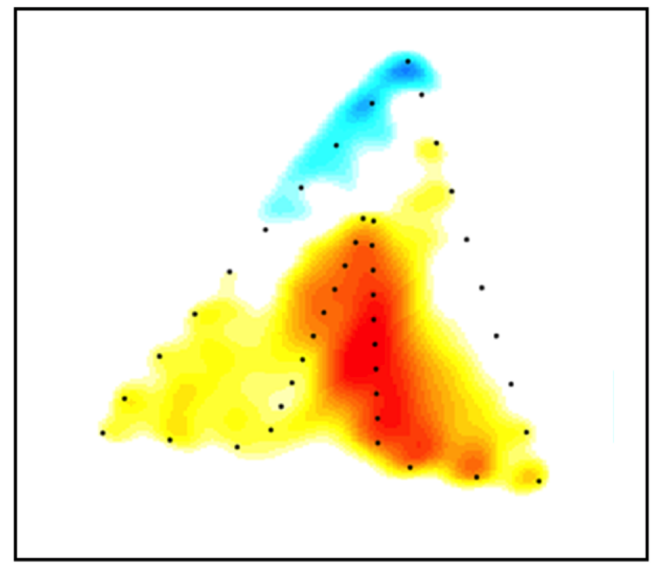

(d) Accounting for non-stationary psd.

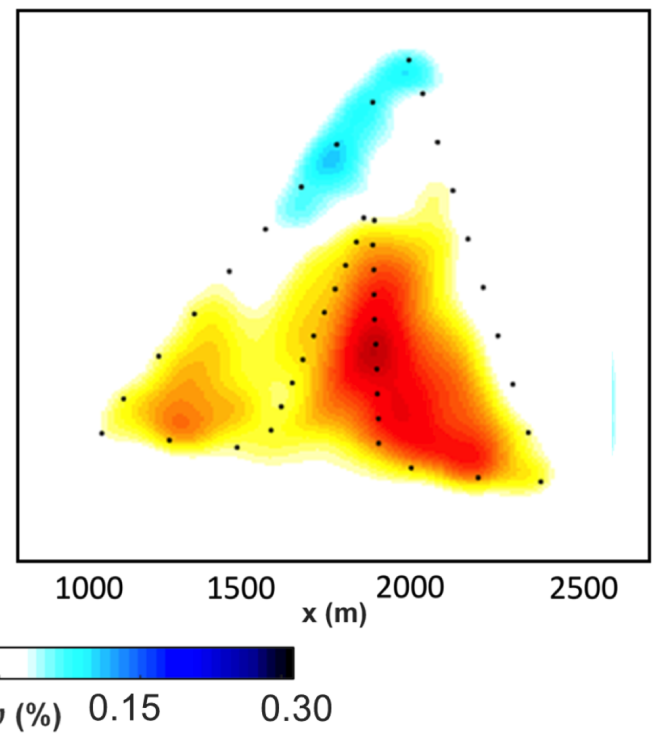

Figure 11: Finite-frequency inversion results attempting to image the true structure perturbation rendered in a). b) Baseline case I, where the non-stationary noise sources are taken into account and assumed to be known perfectly. This is the best possible reconstruction using the given data. c) Case II where we image time-lapse velocity perturbations assuming that noise sources are homogeneous and not variable in time. d) Case III where we do correct for noise source non-stationarity biases by imaging the noise source $p s d$. Correcting for noise source biases allows us to remove spurious artifacts and better identify the true structural change. In case II, it is difficult t 54 argue whether the imaged positive anomaly in the northwestern corner is oenuine. With the correction anplied in case III we see that 


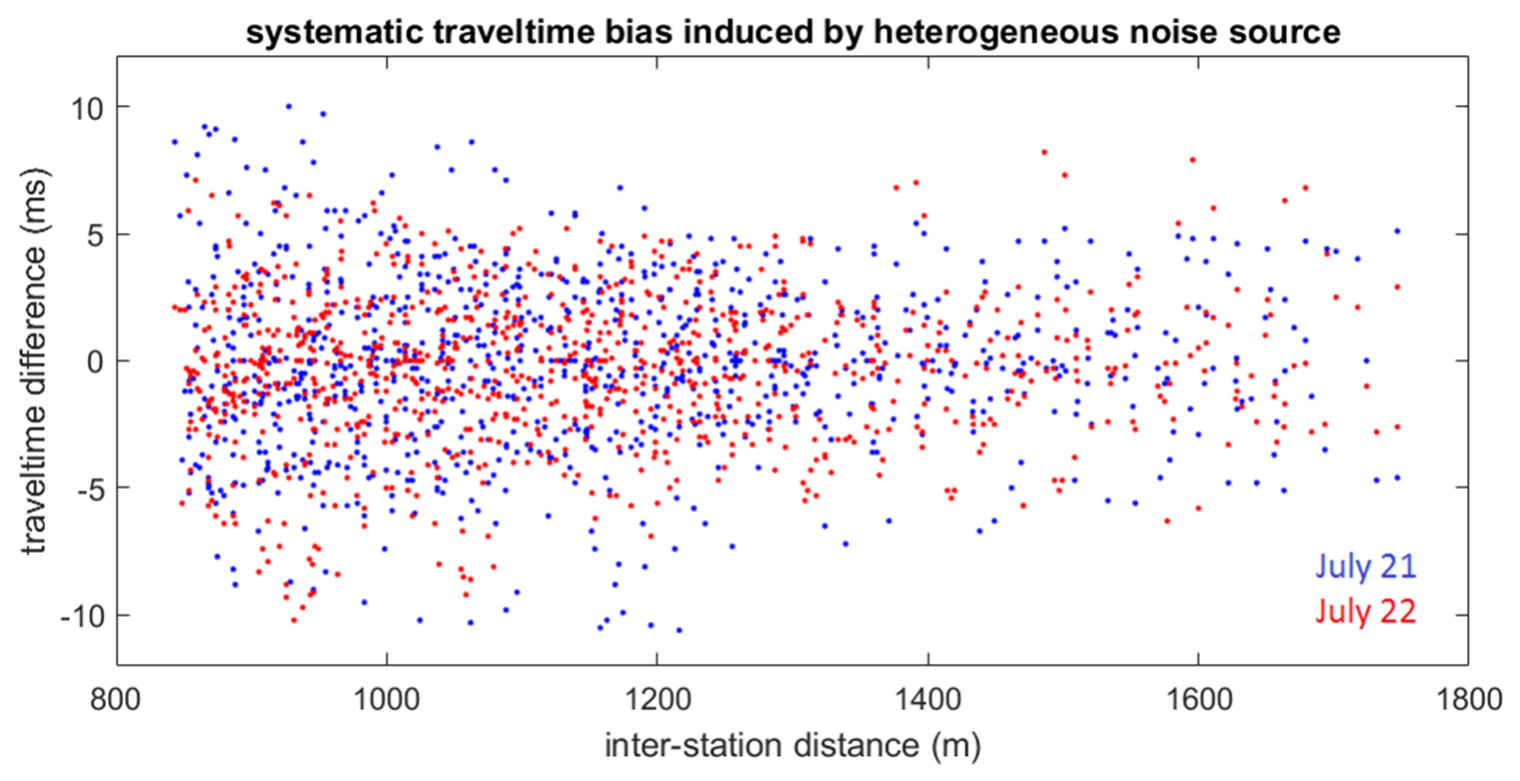

Figure 12: Traveltime differences between synthetic correlations $\bar{C}\left(\mathbf{x}_{i}, \mathbf{x}_{k}, t\right)$ computed for an isotropic noise source distribution and artificial data $\bar{C}^{\mathrm{o}}\left(\mathbf{x}_{i}, \mathbf{x}_{k}, t\right)$ computed for the noise source distributions on July 21 (blue) and July 22 (red), shown in Figures 6 and 7 - now plotted against inter-station pair distance. Systematic errors depend on $p s d$ azimuths with respect to inter-station pair azimuths and are less sensitive to inter-station pair distance. 

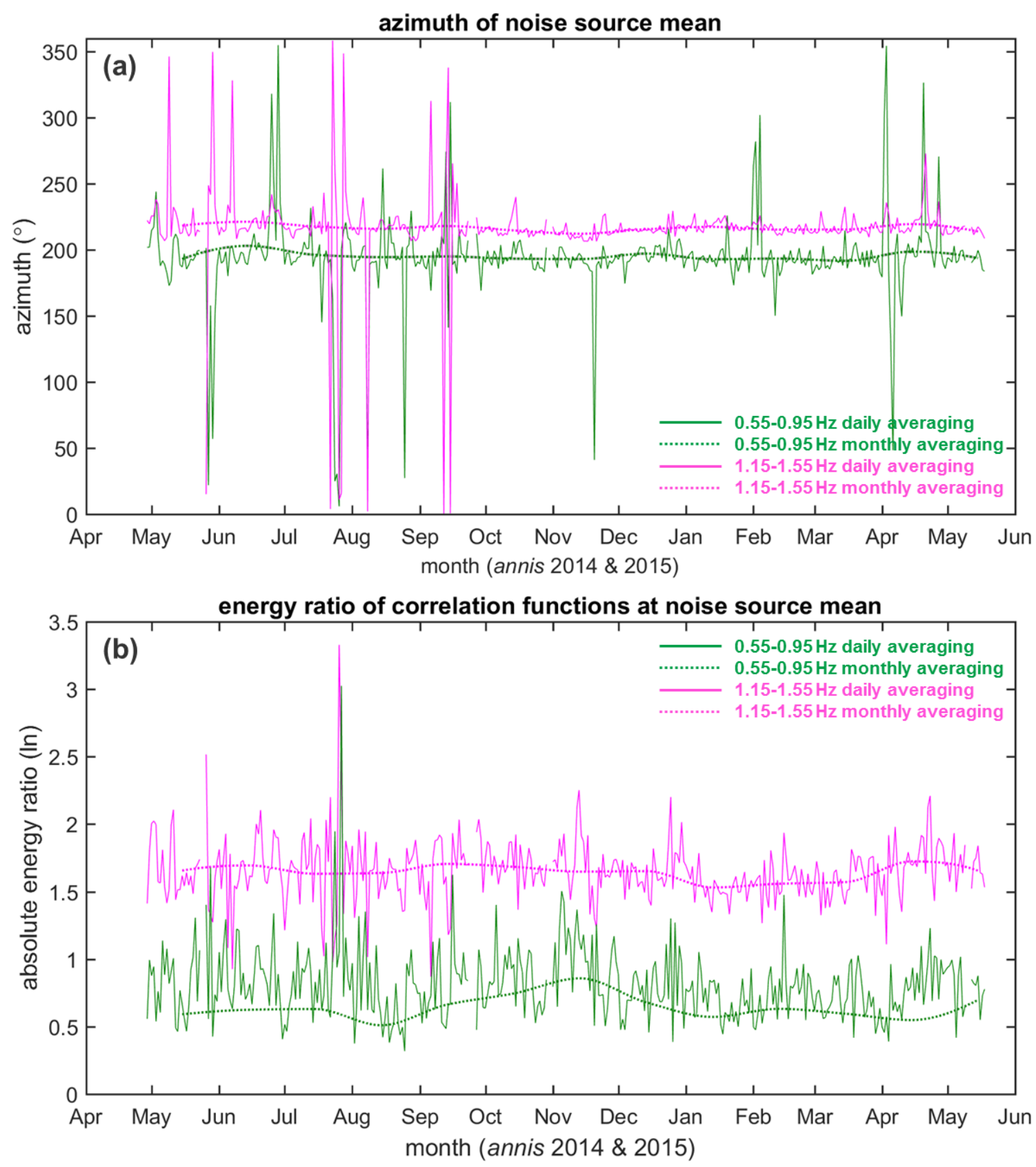

Figure 13: (a) Weighted angular mean of the noise source azimuth as a function of daily averaging (solid line) and of monthly averaging (dotted line) for frequency bands 0.55-0.95 $\mathrm{Hz}$ (green) and 1.15-1.55 Hz (magenta). (b) Mean absolute energy ratio of correlations at corresponding angular mean. As we invert for noise source psd by using energy ratios, we can use the energy ratios as a $p s d$ analogue. We observe that noise source non-stationarity is spatially, frequency, and temporally dependent and that temporal dependence can be 